\title{
Las investigaciones sobre movimientos de laderas en Costa Rica, Honduras, México y Nicaragua: enseñanzas desde la academia, las agencias de cooperación y las instituciones públicas
}

\author{
Research on hillslope processes in Costa Rica, Honduras, \\ Mexico and Nicaragua: teachings from the academy, \\ cooperation agencies and public institutions
}

\author{
Adolfo Quesada-Román \\ University of Geneva, Suiza \\ Rigoberto Moncada-López ${ }^{2}$ \\ Universidad Tecnológica Centroamericana, Honduras \\ Jorge Antonio Paz-Tenorio ${ }^{3}$ \\ Universidad de Ciencias y Artes de Chiapas, México \\ Eveling Espinoza-Jaime ${ }^{4}$ \\ Carmen Gutiérrez-Gutiérrez ${ }^{5}$ \\ Alex Castellón-Meyrat \\ Norwin Acosta-Galeano \\ Instituto Nicaragüense de Estudios Territoriales, Nicaragua
}

http://dx.doi.org/10.15359/rgac.60-1.1

\footnotetext{
Adolfo Quesada Román, University of Geneva. Correo electrónico: adolfo.quesada@gmail.com

2 Rigoberto Moncada López, Universidad Tecnológica Centroamericana. Correo electrónico: rigoberto.moncada@unitec.edu

3 Jorge Antonio Paz Tenorio, Universidad de Ciencias y Artes de Chiapas. Correo electrónico: jorge.paz@unicach.mx

4 Eveling Espinoza Jaime, Instituto Nicaragüense de Estudios Territoriales. Correo electrónico: evelingespinoza@hotmail.com

5 Carmen Gutiérrez Gutiérrez, Instituto Nicaragüense de Estudios Territoriales. Correo electrónico: carmen. gutierrez@gf.ineter.gob.ni

6 Alex Castellón Meyrat, Instituto Nicaragüense de Estudios Territoriales. Correo electrónico: casteyrat@hotmail.com

7 Norwin Acosta Galeano, Instituto Nicaragüense de Estudios Territoriales. Correo electrónico: norwinacosta@hotmail.com
} 
Adolfo Quesada-Román, Rigoberto Moncada-López, Jorge Antonio Paz-Tenorio, Eveling Espinoza-Jaime, Carmen Gutiérrez-Gutiérrez, Alex Castellón-Meyrat, Norwin Acosta-Galeano. Research on hillslope processes in Costa Rica, Honduras, Mexico and Nicaragua: teachings from the academy, cooperation agencies and public institutions

\title{
RESUMEN
}

En este trabajo se especifica la gama de productos a partir de los cuales especialistas de Costa Rica, Honduras, México y Nicaragua desarrollan cartografía sobre movimientos de laderas, aspecto que puede ilustrar la accesibilidad a ciertos datos, así como la escasez o falta de información específica en la región. Trabajos realizados desde Instituciones gubernamentales, Agencias de Cooperación y la Academia, procuran sumar esfuerzos con las autoridades nacionales de cada lugar en el estudio de este fenómeno. Se respeta la terminología utilizada en cada proyecto, por lo que se considera equivalente el término Movimientos de Laderas. Esta cartografía tiene como fin ser un insumo de línea base para estudios de Gestión del Riesgo de Desastres y el Ordenamiento Territorial de ciudades, cuencas y municipios a lo largo de América Latina.

Palabras clave: Movimientos de Ladera; Geomorfología; Mapeo de Deslizamientos; Procesos de Ladera; Susceptibilidad de Deslizamientos.

\begin{abstract}
This paper specifies the range of products from which specialists from Costa Rica, Honduras, Mexico and Nicaragua develop cartography on hillslope movements, an aspect that can illustrate the accessibility to certain data, as well as the scarcity or lack of specific information in the region. Works carried out from Government Institutions, Cooperation Agencies and the Academy try to join forces with national authorities at each place in the study of this phenomenon. The terminology used in each project is respected, and the term hillside movements is considered equivalent. This mapping is intended to be a baseline input for studies on Disaster Risk Management and Land Use Planning in cities, basins and municipalities throughout Latin America.
\end{abstract}

Keywords: Slope movements; Geomorphology; Landslide Mapping; Slope Processes; Landslide Susceptibility.

\section{Introducción}

Los procesos de ladera se presentan en todas las latitudes y altitudes del globo, tienen una vasta variedad de condiciones, así como factores que los detonan, entre ellos, las fuertes lluvias, los sismos y la actividad del ser humano (Anderson et al., 2011; Sterlacchini et al., 2011). A pesar de presentarse sin distinción geográfica, estos fenómenos se dan con mayor dominancia en ambientes fríos (precipitaciones y temperaturas anuales menores a $1.000 \mathrm{~mm}$ y $0{ }^{\circ} \mathrm{C}$, respectivamente) y en zonas templadas a tropicales (precipitaciones y temperaturas anuales mayores a $1.700 \mathrm{~mm}$ y $0{ }^{\circ} \mathrm{C}$ ) (Selby, 1985). En términos de desastres, los movimientos en masa varían de magnitudes bajas a altas, ocurren con una relativa frecuencia, son de poca duración, y su extensión areal, por lo general, es limitada (Gares et al., 1994). Las causas que generan los procesos de ladera se podrían dividir en naturales y humanas, donde las primeras incluyen al agua, la actividad sísmica y la actividad volcánica, cada uno como un elemento 
Adolfo Quesada-Román, Rigoberto Moncada-López, Jorge Antonio Paz-Tenorio, Eveling Espinoza-Jaime,

Carmen Gutiérrez-Gutiérrez, Alex Castellón-Meyrat, Norwin Acosta-Galeano. Las investigaciones sobre movimientos de laderas en Costa Rica, Honduras, México y Nicaragua: enseñanzas desde la academia, las agencias de cooperación y las instituciones públicas

individual o en combinación; las causas humanas o antrópicas van desde la deforestación, la urbanización, la ganadería y la agricultura, que modifican el ángulo de reposo de las laderas y propician su inestabilidad (Highland y Bobrowsky, 2008).

Los términos para nombrar a los procesos que se dan en las laderas y, que están regidos por la gravedad, derivan del inglés, la traducción al español a veces genera confusión, por lo que reconoce varias formas: procesos de ladera (slope processes), procesos gravitacionales (gravitational processes), procesos de remoción en masa (mass movement processes) o deslizamientos (landslides); todos ellos correctos (Alcántara-Ayala, 2000). Los diferentes procesos de ladera se ordenan según las siguientes clases (UNESCO Working Partyon World Landslide Inventory (WP/WLI 1990); Cruden y Varnes, 1996): Deslizamientos, Caídas o Desprendimientos, Volteos, Flujos, Expansión lateral, Movimientos complejos. Los materiales que componen los tipos de procesos de remoción en masa se dividen según el tipo de material en: roca, detritos (partículas mayores a $2 \mathrm{~mm}$ ) y suelos (partículas menores a $2 \mathrm{~mm}$ ).

El objetivo de este trabajo es conocer las metodologías aplicadas en cuatro países de América Latina (Costa Rica, Honduras, México y Nicaragua) y cómo dichos procedimientos pueden generar mapas de susceptibilidad de movimientos de ladera, los cuales sirven para gestionar los riesgos y ordenar el territorio de distintas ciudades, cuencas o unidades político-administrativas a partir de los esfuerzos de la academia, agencias de cooperación o las instituciones públicas. Por tanto, se parte de la caracterización de cada una de las zonas de estudio, posteriormente, se hace una explicación por caso de estudio para entender las distintas metodologías utilizadas en la cartografía de movimientos de ladera en cuatro casos de Costa Rica, Honduras, México y Nicaragua; se presentan sus resultados, se hace una discusión de las fortalezas y debilidades de cada una de estas metodologías, así como los estudios de movimientos de ladera en cada uno de estos países y el alcance que tienen en la gestión del riesgo de desastres.

\section{Caso de Costa Rica: la cuenca alta del río General}

En países tropicales como Costa Rica, la incidencia de procesos de ladera es asociada a la meteorización, el tipo de sustrato, la sismicidad, la morfología y la inclinación del terreno. La recurrencia y magnitud de 
Adolfo Quesada-Román, Rigoberto Moncada-López, Jorge Antonio Paz-Tenorio, Eveling Espinoza-Jaime, Carmen Gutiérrez-Gutiérrez, Alex Castellón-Meyrat, Norwin Acosta-Galeano. Research on hillslope processes in Costa Rica, Honduras, Mexico and Nicaragua: teachings from the academy, cooperation agencies and public institutions

estos procesos afectan en términos de pérdidas económicas y muertes en las comunidades de la cuenca alta del río General en el centro-sur del país. En el pasado, eventos como el sismo de Buenavista (1983), los huracanes Joan (1988), César (1996) y la tormenta tropical Alma (2008) causaron pérdidas humanas y graves daños económicos en líneas vitales (vías de comunicación, cableado eléctrico y acueductos), así como en el sector agropecuario (Quesada-Román, 2017).

La cuenca alta del río General se localiza entre los $9^{\circ} 12^{\prime} 46^{\prime \prime}-9^{\circ} 35^{\prime}$ 03 " de latitud N y los $83^{\circ} 28^{\prime} 47^{\prime \prime}$ - 83 49'07" de longitud W. El relieve de este territorio es resultado de una dinámica tectónica compleja, que tiene relación con los movimientos regionales del fondo oceánico y del continente. En esta geodinámica se vinculan varias placas tectónicas en un proceso de subducción entre Cocos y Caribe, la colisión de la Cordillera submarina de Cocos (Cocos Ridge), la junta triple entre Placas Cocos, Caribe y Nazca, y fallas activas (transcurrentes) asociadas a la Placa Panamá (Bird, 2003; Denyer et al., 2003). Otras de las variables que hacen particular este territorio son la precipitación, la vegetación y los usos de la tierra. La cuenca alta del río General posee vínculo directo entre las zonas de mayor precipitación (entre 4.000 y $5.500 \mathrm{~mm}$ anuales) con amplias regiones de laderas que son modeladas por la actividad gravitacional, así como las vertientes afectadas por la reptación y la arroyada. Los usos de la tierra en el área de estudio corresponden a zonas agrícolas de café, pastos, uso forestal y vegetación de páramo (Quesada-Román, 2016).

\section{Metodología}

Para poder desarrollar una cartografía precisa en la zonificación de peligros geomorfológicos, como los procesos de ladera, es importante desarrollar análisis del relieve basados en la morfometría y la morfogénesis (geomorfología). La morfometría es la parte de la geomorfología que estudia las características cuantitativas de las formas de relieve a partir de parámetros cartográficos; cálculo de áreas, volumen, inclinación del terreno, densidad de alguna forma terrestre sobre una superficie determinada, para establecer una relación entre los procesos de modelado y la litología (Lugo-Hubp, 2011). Se reconoce que la morfometría es un importante componente en el estudio del terreno, sobre todo, cuando se habla del modelado de la superficie terrestre (Huggett, 2007). Los productos que resultan de 
este tipo de análisis son mapas, cada uno de ellos refleja la espacialidad de la medición realizada en las formas de relieve analizadas y son necesarios para detectar terrenos susceptibles a presentar dinámicas que pueden ser de peligro (Peña-Monné, 1997).

La geomorfología, por su parte, es la ciencia que estudia el relieve y en ello toma en cuenta génesis, morfología, dinámica, evolución y edad. La cartografía geomorfológica es parte de este análisis y tiene como objetivo representar, con límites precisos, a partir de una simbología gráfica propia de las ciencias de la tierra, la arquitectura de la superficie terrestre, por tanto, el mapa geomorfológico a través de un lenguaje gráfico muestra la génesis del relieve, su litología, el arreglo estructural, la cronología y la morfología de cualquier territorio (Quesada-Román, 2016). El mapa de peligros geomorfológicos resume el análisis morfométrico y morfogenético en una clasificación de las zonas con distintos niveles de susceptibilidad a procesos de ladera e inundaciones, terrenos diferenciados en su explicación debido a que tienen agentes disparadores, extensiones espaciales y dinámicas distintas a partir de sus características geomorfológicas.

\section{Resultados}

De acuerdo a Quesada-Román (2016), se describen las condiciones que favorecen la existencia de terrenos susceptibles a presentar diferentes grados de afectación real por procesos de remoción en masa. Se establecieron tres categorías cuya representación espacial se identifica con el color rojo las zonas de máxima ocurrencia, con el color naranja de baja ocurrencia y el color amarillo representa áreas potenciales (Figura 1). 
Adolfo Quesada-Román, Rigoberto Moncada-López, Jorge Antonio Paz-Tenorio, Eveling Espinoza-Jaime, Carmen Gutiérrez-Gutiérrez, Alex Castellón-Meyrat, Norwin Acosta-Galeano. Research on hillslope processes in Costa Rica, Honduras, Mexico and Nicaragua: teachings from the academy, cooperation agencies and public institutions

Figura 1. Mapa de peligros a procesos de ladera de la cuenca alta del río General

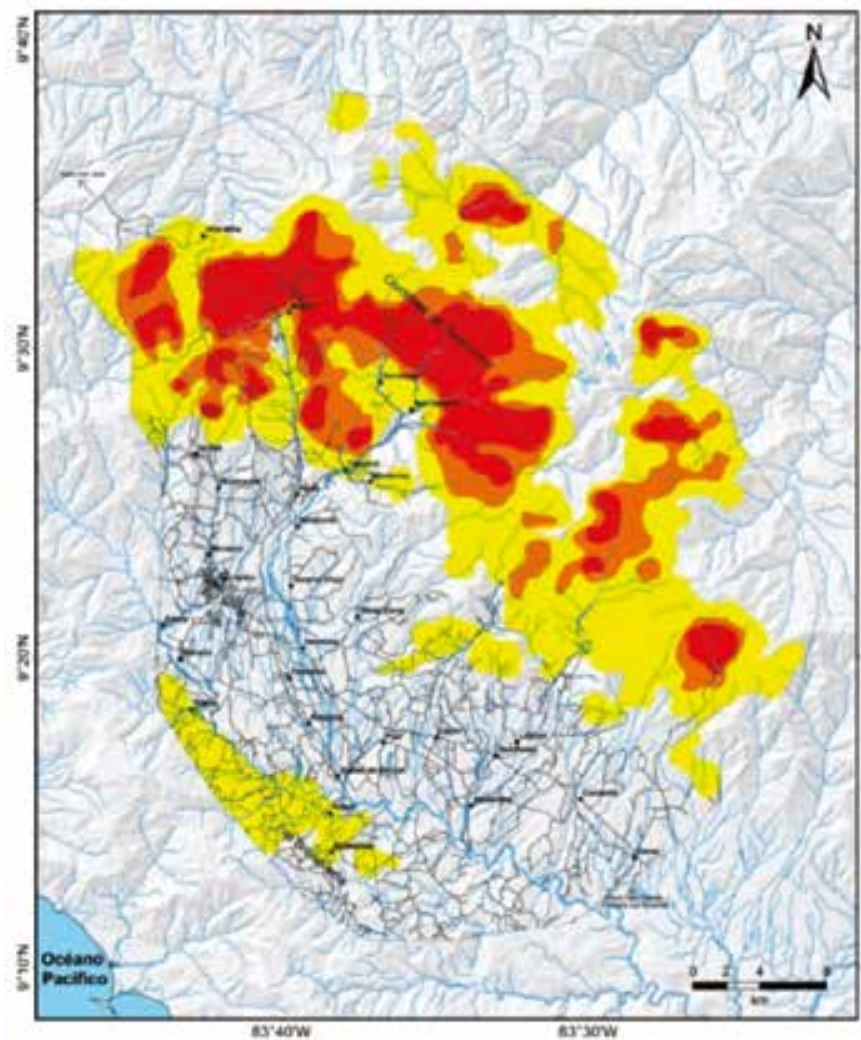

\section{Leyenda}

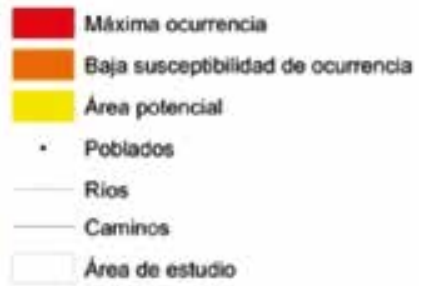

Fuente: Quesada-Romaln (2016) GCS / WGS 84

Los sitios de máxima ocurrencia a procesos de ladera se localizan en las cabeceras de los ríos Chirripó y Telire al NE, y los ríos Savegre (hacia el NW), Buenavista y Chirripó Pacífico (al N), así como el Peñas Blancas, San Pedro y Volcán (hacia el SE), terrenos que suman un total de 130 $\mathrm{km}^{2}$, equivalente al $8 \%$ de la superficie de estudio. Tienen inclinaciones superiores a $25^{\circ}$, donde los afloramientos geológicos típicos son de rocas volcánicas y plutónicas del Mioceno en morfologías de laderas cóncavas y rectas. Los terrenos de máxima ocurrencia a procesos de ladera son activados por las lluvias estacionales ( $>2500 \mathrm{~mm}$ anuales) y ciclónicas (entre agosto y noviembre), además por la influencia sismo-tectónica (terremotos interplaca e intraplaca). De acuerdo al análisis geomorfológico en estas regiones se cartografiaron 21 deslizamientos, 307 desprendimientos, 12 
Adolfo Quesada-Román, Rigoberto Moncada-López, Jorge Antonio Paz-Tenorio, Eveling Espinoza-Jaime, Carmen Gutiérrez-Gutiérrez, Alex Castellón-Meyrat, Norwin Acosta-Galeano. Las investigaciones sobre movimientos de laderas en Costa Rica, Honduras, México y Nicaragua: enseñanzas desde la academia, las agencias de cooperación y las instituciones públicas

flujos de rocas y 9 flujos de lodo. De acuerdo a los registros de desastres DesInventar (LA RED, 2015), entre 1980 y 2010 el 16,4\% de los procesos de ladera reportados coinciden con estos sitios.

Los terrenos susceptibles a procesos de ladera de baja ocurrencia se definen como extensas áreas que encapsulan las regiones de máxima ocurrencia, donde su localización se da en las cabeceras de las cuencas de los ríos Chirripó y Telire (vertiente Caribe) y los ríos Savegre, Buenavista, Chirripó Pacífico, San Pedro y Volcán (vertiente Pacífica), equivalente a un 9,35\% del territorio. Estos terrenos se desarrollan en laderas de montaña donde predominan las morfologías cóncavas y rectas con inclinaciones entre $16^{\circ}$ y $63^{\circ}$, situados en sustratos de rocas granitoides del Mioceno con edades entre 11 y $6 \mathrm{Ma}$ (Denyer y Alvarado, 2007). Se podrían producir deslizamientos, flujos de roca, flujos de lodo, caídas de rocas y desprendimientos; procesos que son detonados por la pluviosidad estacional superior a $2500 \mathrm{~mm}$ anuales, así como las lluvias ciclónicas presentes entre agosto y octubre, además de episodios sísmicos debido a varias fallas activas locales y la cercanía con la Trinchera Mesoamericana. En estas zonas fueron identificados mediante el mapa geomorfológico 44 deslizamientos, 275 desprendimientos, 18 flujos de rocas y 9 flujos de lodo.

Las Áreas potenciales a procesos de ladera se localizan cerca de poblados como División, San Juan Norte, San Gerardo de Rivas, Esperanzas y Mollejones; tienen una extensión territorial de $403 \mathrm{~km}^{2}$, lo que equivale a un $25,8 \%$ del área de estudio. Se ubican en laderas de montaña rectas y cóncavas con pendientes que son menores a $25^{\circ}$, sobre sustratos volcánicos, plutónicos y sedimentarios del Mioceno con edades entre 29 y 2 Ma (Denyer y Alvarado, 2007). En estas regiones se cartografiaron 73 deslizamientos, 333 desprendimientos, 47 flujos de rocas y 14 flujos de lodo, y según la base de datos de desastres DesInventar (LA RED, 2015) entre 1980 y 2010, un 37,7\% del total (61 registros) se ubica dentro de estas superficies. Estos terrenos han evolucionado en el tiempo dadas la intensa precipitación (mayor a $2500 \mathrm{~mm}$ anuales), la actividad tectónica y el fallamiento activo, además en la actualidad se presenta una modificación antrópica de bosques hacia usos agropecuarios que favorecen el incremento de dichas superficies. Por último, los terrenos con estabilidad aparente a procesos de ladera suman un 64\% $\left(1002 \mathrm{~km}^{2}\right)$ del área de estudio, donde la incidencia de procesos de ladera es mínima o nula debido en buena medida a la estabilidad de los materiales, donde las pendientes por lo general se mantienen en los rangos medios o bajos (menores a $25^{\circ}$ ). 
Adolfo Quesada-Román, Rigoberto Moncada-López, Jorge Antonio Paz-Tenorio, Eveling Espinoza-Jaime, Carmen Gutiérrez-Gutiérrez, Alex Castellón-Meyrat, Norwin Acosta-Galeano. Research on hillslope processes in Costa Rica, Honduras, Mexico and Nicaragua: teachings from the academy, cooperation agencies and public institutions

\section{Los estudios de movimientos de ladera en Costa Rica}

Para realizar un análisis de los procesos de ladera que se han dado en algún territorio de Costa Rica es necesario hacer uso, como primer insumo y acercamiento al historial, de la base de datos DesInventar. La información de base para generar estudios de movimientos en masa en Costa Rica son los insumos que tiene el Instituto Geográfico Nacional con las curvas de nivel a escala 1:50.000 que se obtienen de las 136 hojas topográficas que conforman el país. En ciertos sectores se cuenta también con curvas de nivel escala 1:25.000, 1:10.000 o incluso mejores. Además, se cuenta con fotografías aéreas desde la década de 1940 hasta la actualidad a diversas escalas disponibles, esta información está en venta o se puede facilitar bajo convenio con ciertas instituciones públicas y académicas tanto desde el país como del exterior. Durante el 2005, se realizó un levantamiento a escala nacional de imágenes 1:25.000 y en algunos sectores urbanos a 1:5.000 por medios del proyecto CARTA, el cual fue integrado por las cuatro universidades estatales (UCR, UNA, UNED, ITCR), el Centro Nacional de Alta Tecnología (CeNAT), la Fundación Costa Rica Estados Unidos para la Cooperación, el Instituto Costarricense de Electricidad y el proyecto BID-CATASTRO. En la actualidad estas fotografías aéreas y otros insumos satelitales están a disposición del PRIAS (Programa de Investigaciones Aerotransportadas y Sensores Remotos) del CeNAT para su venta al público en general y la información puede ser solicitada de manera gratuita bajo convenio entre ciertas instancias y objetivos de investigación. En este momento el Catastro Nacional está realizando una actualización de su información para todo el país, la cual podría ser utilizada para fines varios, como la actualización registral, los cambios en el uso del suelo o incluso el desarrollo de mapas de vegetación, geomorfológicos y otras aplicaciones, dadas las nuevas tendencias en la precisión de los insumos fotogramétricos.

Los encargados oficiales de generar y avalar la cartografía de procesos de ladera, desde su fundación en 1969 ha sido la Comisión Nacional de Emergencias (CNE), quien ha hecho uso del conocimiento de diversos expertos en Geología, Geografía, Ingeniería Civil, Geomorfología y otras ciencias afines que trabajan directamente para esta institución, o del apoyo o estudios de otras instituciones públicas como el Instituto Costarricense de Electricidad (ICE), Acueductos y Alcantarillados (AyA), y diversos 
Adolfo Quesada-Román, Rigoberto Moncada-López, Jorge Antonio Paz-Tenorio, Eveling Espinoza-Jaime,

Carmen Gutiérrez-Gutiérrez, Alex Castellón-Meyrat, Norwin Acosta-Galeano. Las investigaciones sobre movimientos de laderas en Costa Rica, Honduras, México y Nicaragua: enseñanzas desde la academia, las agencias de cooperación y las instituciones públicas

ministerios como el Ministerio de Ambiente (MINAE), el Ministerio de Obras Públicas y Transportes (MOPT), entre otros. Además, de mucha valía ha sido el apoyo de la academia desde distintas escuelas y centros de investigación de la Universidad de Costa Rica (Escuela Centroamericana de Geología, Escuela de Geografía, Escuela de Ingeniería Civil y otras; Red Sismológica Nacional UCR-ICE; LANAMME-UCR), Universidad Nacional de Costa Rica (Observatorio Vulcanológico y Sismológico de Costa Rica-UNA, Escuela de Ciencias Geográficas, y otros), Instituto Tecnológico de Costa Rica y la Universidad Estatal a Distancia, así como de consultores nacionales e internacionales para levantar los mapas de movimientos en masa a diversas escalas dependiendo del objetivo de cada estudio.

Los mapas que están avalados por la CNE deben ser mejorados más allá de la escala 1:50.000 que actualmente gozan todos los cantones del país y generar mejores estudios, a escalas detalladas (1:25.000, 1:10.000 o superiores) de los procesos de ladera que afectan el territorio nacional. Estos estudios deben ser avalados por la CNE y ser generados del vínculo con las universidades públicas y expertos en la temática. Dentro de esta misma línea, se debe procurar generar más estudios sobre multiamenazas ligados con análisis probabilísticos de acuerdo a las características particulares de las cuencas que integran el país, ya que los movimientos en masa normalmente no ocurren como procesos aislados y su estudio debe abordarse desde la Gestión del Riesgo y el Ordenamiento Territorial.

\section{Caso de Honduras: Mapa de Inventario de Deslizamientos de Tierra en Base a Interpretación de Fotografías Aéreas}

El área de estudio en mención es la ciudad capital de Honduras en la zona metropolitana de Tegucigalpa, Comayagüela y alrededores dentro del municipio del Distrito Central, departamento de Francisco Morazán. En los últimos 20 años, Honduras ha tenido el mayor índice de riesgo climático y es considerado el país más afectado por catástrofes naturales junto a Myanmar y Haití (Kreft et al., 2016). Factores comunes en Latinoamérica, como el crecimiento acelerado y sin orden en los centros de población, inciden directamente en la ocurrencia de desastres como inundaciones y deslizamientos de tierra. Ocasionalmente, también ha habido repercusiones por fenómenos sísmicos. Desde el punto de vista de los deslizamientos, algunos de los fenómenos naturales que han generado 
Adolfo Quesada-Román, Rigoberto Moncada-López, Jorge Antonio Paz-Tenorio, Eveling Espinoza-Jaime, Carmen Gutiérrez-Gutiérrez, Alex Castellón-Meyrat, Norwin Acosta-Galeano. Research on hillslope processes in Costa Rica, Honduras, Mexico and Nicaragua: teachings from the academy, cooperation agencies and public institutions

mayor cantidad de movimientos de ladera han sido el Huracán Fifí en 1974, el Huracán Gilbert en 1988, el Huracán Mitch en 1998 (Harp et al. 2002), la tormenta tropical 16 en 2008 y el terremoto de magnitud 7.3 del 28 de marzo de 2009 (PNUD, 2010). La mayor parte de los deslizamientos han sido atribuidos a la detonación por precipitaciones intensas, este ha sido el enfoque principal para los análisis de amenaza y susceptibilidad hacia los movimientos superficiales de ladera, acompañados por unos pocos deslizamientos a gran escala de impacto considerable (Harp et al. 2002) (JICA, 2002). En este contexto se ha identificado la importancia de variables geológicas y topográficas en la generación de estos movimientos (PNUD, 2010), aunadas a un crecimiento poblacional acelerado que afecta la cobertura y topografía de los terrenos, causando un aumento en la vulnerabilidad de los habitantes (Ebert \& Kerle, 2008).

El lugar de estudio combina la topografía, tipo valle de la cuenca alta del Río Choluteca, y donde solía estar localizada la mayor parte de la ciudad desde 1578 hasta su crecimiento durante el siglo XX, con una topografía montañosa accidentada en su periferia, sus elevaciones van desde 900 hasta, aproximadamente, $1300 \mathrm{msnm}$ (PNUD, 2010). El área completa de la ciudad se encuentra entre dos subcuencas de la cuenca principal del Río Choluteca: Choluteca Alta y Guacerique-Río Grande. El Río Choluteca tiene su naciente cerca de Tegucigalpa y desemboca en el océano Pacífico. Es justo dentro de la zona urbana de Tegucigalpa, que numerosos de sus afluentes se combinan para formar el río principal, y la ciudad es atravesada de norte a sur casi por completo por su cauce (IHCIT, 2014). Casi la totalidad de los bordes del Río, ubicados dentro de la zona urbana, están ocupados por asentamientos.

La precipitación tiene un valor promedio anual entre 1075-1175 mm (IHCIT, 2012), aunque en el caso del Huracán Mitch se alcanzaron valores de $281 \mathrm{~mm}$ en cerca de una semana (Harp et al., 2009). Históricamente, los meses de mayo, junio, septiembre y octubre son aquellos con las máximas precipitaciones, alcanzando valores máximos diarios cercanos a los 130 $\mathrm{mm}$. La correlación entre ocurrencia de movimientos de ladera y precipitación ha sido demostrada para diferentes estaciones meteorológicas en la zona (IHCIT, 2011).

Geológicamente, los principales grupos o formaciones son de origen volcánico y sedimentario. El Grupo Padre Miguel, el cual cubre el mayor 
Adolfo Quesada-Román, Rigoberto Moncada-López, Jorge Antonio Paz-Tenorio, Eveling Espinoza-Jaime,

Carmen Gutiérrez-Gutiérrez, Alex Castellón-Meyrat, Norwin Acosta-Galeano. Las investigaciones sobre movimientos de laderas en Costa Rica, Honduras, México y Nicaragua: enseñanzas desde la academia, las agencias de cooperación y las instituciones públicas

porcentaje del sitio de estudio, consta de tobas ríolíticas, dacíticas y andesíticas, lahares, ignimbritas y otros sedimentos piroclásticos del Terciario (IHCIT, 2014). Otras unidades volcánicas en la zona son la formación Matagalpa, que se compone de coladas máficas de basalto y andesitas, de posición estratigráfica pre-ignimbrítica y también del Terciario; y coladas basálticas oscuras del Cuaternario (Rogers, 1993). Por otro lado, el Grupo Valle de Ángeles, el cual aflora en la zona centro, este y sureste de la ciudad, se presenta con sus formaciones Villanueva (conglomerados) y Río Chiquito (lutitas y areniscas) (PNUD, 2010). Y en terrazas en zonas bajas o fluviales y pies de ladera se encuentran depósitos de aluviones del Cuaternario (IGN, 1987).

\section{Metodología}

Dentro del marco de proyecto "Hazard Geology Focusingon Landslides in Tegucigalpa", desarrollado por investigadores de la Agencia Internacional de Cooperación del Japón (JICA) con fondos de Sociedad Japonesa para la Promoción de las Ciencias (JSPS), entre 2012 y 2014, se propuso desarrollar un mapa actualizado del inventario de deslizamientos de tierra o movimientos de ladera (Yamagishi et al., 2014).

En esencia, se determinaron sus ubicaciones, perímetros y áreas y se estableció una clasificación general en función de la fotointerpretación para identificar dichos deslizamientos. El área de trabajo fue la zona metropolitana de Tegucigalpa, Comayagüela y alrededores, usando como información de base fotografías aéreas tomadas en febrero de 2013 y donadas también por JICA. El estudio cubrió extensiones de aproximadamente 300 $\mathrm{km}^{2}$ para el análisis realizado por los expertos (Yamagishi, 2014; Sato, 2015) y de $400 \mathrm{~km}^{2}$ para aquel desarrollado por un grupo de estudiantes universitarios capacitados con la misma metodología y asesorados por los expertos (TLMG, 2014).

La herramienta principal de trabajo fue la aplicación de la fotogeología e interpretación de imágenes aéreas apoyadas en el uso de la estereoscopía. Las imágenes utilizadas fueron capturadas en una escala de 1:9.000 y junto con estereoscopios de espejo, equipados con lentes de aumento, fue posible visualizar de forma tridimensional y con la claridad suficiente la topografía de sitios con la geomorfología y característica de movimientos de ladera. La clasificación propuesta por Varnes (1978) y 
Adolfo Quesada-Román, Rigoberto Moncada-López, Jorge Antonio Paz-Tenorio, Eveling Espinoza-Jaime, Carmen Gutiérrez-Gutiérrez, Alex Castellón-Meyrat, Norwin Acosta-Galeano. Research on hillslope processes in Costa Rica, Honduras, Mexico and Nicaragua: teachings from the academy, cooperation agencies and public institutions

su actualización desarrollada por Hungr et al. (2013) se emplearon como guías para facilitar la identificación de formas en el terreno que revelasen indicios de deslizamientos ocurridos en el pasado en la zona de estudio.

En varios casos, estos sitios identificados en las fotografías, también fueron corroborados y caracterizados mediante visitas de campo, aunque dicha labor no se ha realizado hasta el momento de forma completa para toda la zona de estudio. Debido a la considerable cantidad de sitios identificados, cerca de 3000 objetos entre todas las capas de información, (Yamagishi, 2014) fue muy conveniente el uso de tecnologías de sistemas de información geográfica (SIG). Se desarrolló un mapa digital de inventario, en donde se incluyeron diferentes signos de movimientos de ladera, tales como: cuerpos de deslizamientos, escarpes, grietas o fisuras, reptación en roca y derrumbes.

Otra información y herramientas de importancia para el desarrollo del mapa incluyeron las hojas cartográficas de Tegucigalpa en escala 1:12.000, los mapas geológicos de Tegucigalpa, Lepaterique y San Buenaventura en escala 1:50.000 (IGN, 1987) y la revisión de perfiles y modelos de terreno tridimensional de Google Earth, los cuales pueden revelar detalles adicionales de deslizamientos (Yamagishi\& Moncada, 2017).

\section{Resultados}

La Figura 2, muestra el mapa completo desarrollado por el equipo de expertos; el grupo de estudiantes también desarrolló su propio mapa por separado. Ambos mapas fueron entregados de forma física (mapas y manual impreso) y digital (formato shape) a autoridades correspondientes en el tema de desastres y planificación urbana. Actualmente, esta información está disponible al público mediante el siguiente portal web: amdc. giscloud.com (AMDC, 2017). 
Adolfo Quesada-Román, Rigoberto Moncada-López, Jorge Antonio Paz-Tenorio, Eveling Espinoza-Jaime,

Carmen Gutiérrez-Gutiérrez, Alex Castellón-Meyrat, Norwin Acosta-Galeano. Las investigaciones sobre movimientos de laderas en Costa Rica, Honduras, México y Nicaragua: enseñanzas desde la academia, las agencias de cooperación y las instituciones públicas

Figura 2. Inventario de Movimientos de Ladera de Tegucigalpa MAPA DE DESLIZAMIENTOS DE TIERRA DE TEGUCIGALPA Versión 2014

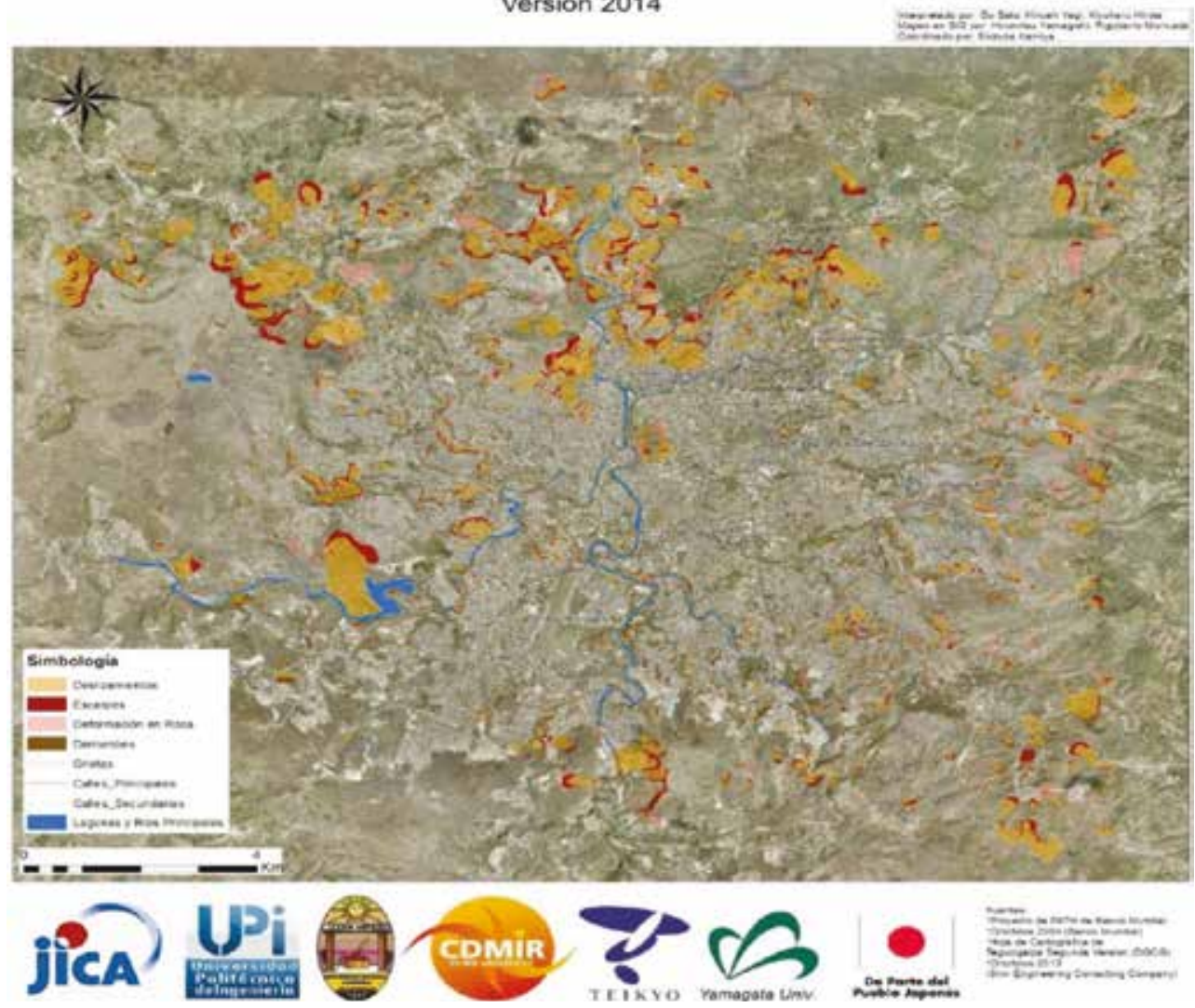

Fuente: Yamagishi et al. (2014)

Al poder trabajar con una escala de 1:9.000 fue posible hacer un inventario que tuvo como resultado la identificación de movimientos de ladera con áreas tan grandes como $0.94 \mathrm{~km} 2 \mathrm{y}$ tan reducidas como $0,000086 \mathrm{~km} 2\left(86 \mathrm{~m}^{2}\right)$, de manera que se pudieron localizar no solamente bloques principales, sino también sub-bloques y derrumbes menores. Para el área de estudio de expertos (300 $\left.\mathrm{km}^{2}\right)$, un $8.43 \%\left(25.28 \mathrm{~km}^{2}\right)$ estaba ocupada por fenómenos de movimientos de ladera. Es importante mencionar que la resolución de la imagen y la percepción de profundidad del estereoscopio pueden ayudar a una caracterización general del deslizamiento, pero se requieren de estudios de campo para clasificar correctamente el tipo movimiento de ladera y su nivel de amenaza. 
Adolfo Quesada-Román, Rigoberto Moncada-López, Jorge Antonio Paz-Tenorio, Eveling Espinoza-Jaime, Carmen Gutiérrez-Gutiérrez, Alex Castellón-Meyrat, Norwin Acosta-Galeano. Research on hillslope processes in Costa Rica, Honduras, Mexico and Nicaragua: teachings from the academy, cooperation agencies and public institutions

En base a los resultados, se determinó que la mayor parte de los deslizamientos se encuentran al norte del área metropolitana, en especial al noroeste. Este hecho se relaciona con la topografía de la ciudad, donde la zona norte presenta mayor cantidad de montañas y pendientes pronunciadas. Si se compara con el mapa multi-amenaza de PNUD/GOAL (CI AMBIENTAL, 2013) que emplea una clasificación por pendientes como uno de sus parámetros, se encuentra un resultado muy similar. De acuerdo a Carías et al. (2015), los mayores valores de disección vertical también se presentan en la mitad norte de Tegucigalpa y muchos de ellos coinciden con la localización de deslizamientos. Asimismo, estas condiciones se combinan con la cercanía al Río Choluteca, donde las zonas con pendiente más pronunciadas resultan en la aglomeración de deslizamientos a lo largo del cauce del río.

Otro factor relevante que puede explicar esta mayor densidad de deslizamientos en el lado norte es la presencia de contactos litológicos como en la frontera entre las rocas volcánicas del Grupo Padre Miguel y las rocas sedimentarias subyacentes del Grupo Valle de Ángeles, en zonas de deslizamientos relevantes, tales como El Berrinche, El Reparto, Col. Viera, Col. Canaán, entre otras. Otro ejemplo de los efectos de estos contactos, se presenta en el Cerro El Pedregal, donde se han identificado y desarrollado numerosos movimientos de ladera en los límites entre un domo de basalto del Cuaternario e ignimbritas y coladas ríolitícas del Grupo Padre Miguel y de la Formación Matagalpa (Hirota\&Kamiya, 2014). En la Figura 3, se muestran ejemplos de estos contactos o fronteras.

Por otro lado, en la zona sur de la ciudad, al tener una topografía predominantemente llana, se encontró menor prevalencia de topografías de deslizamientos. Lo mismo puede atribuirse a la menor cantidad de contactos litológicos, en comparación a la zona norte. Aun así, también se presentaron numerosos movimientos de ladera en ciertas zonas. 
Adolfo Quesada-Román, Rigoberto Moncada-López, Jorge Antonio Paz-Tenorio, Eveling Espinoza-Jaime, Carmen Gutiérrez-Gutiérrez, Alex Castellón-Meyrat, Norwin Acosta-Galeano. Las investigaciones sobre movimientos de laderas en Costa Rica, Honduras, México y Nicaragua: enseñanzas desde la academia, las agencias de cooperación y las instituciones públicas

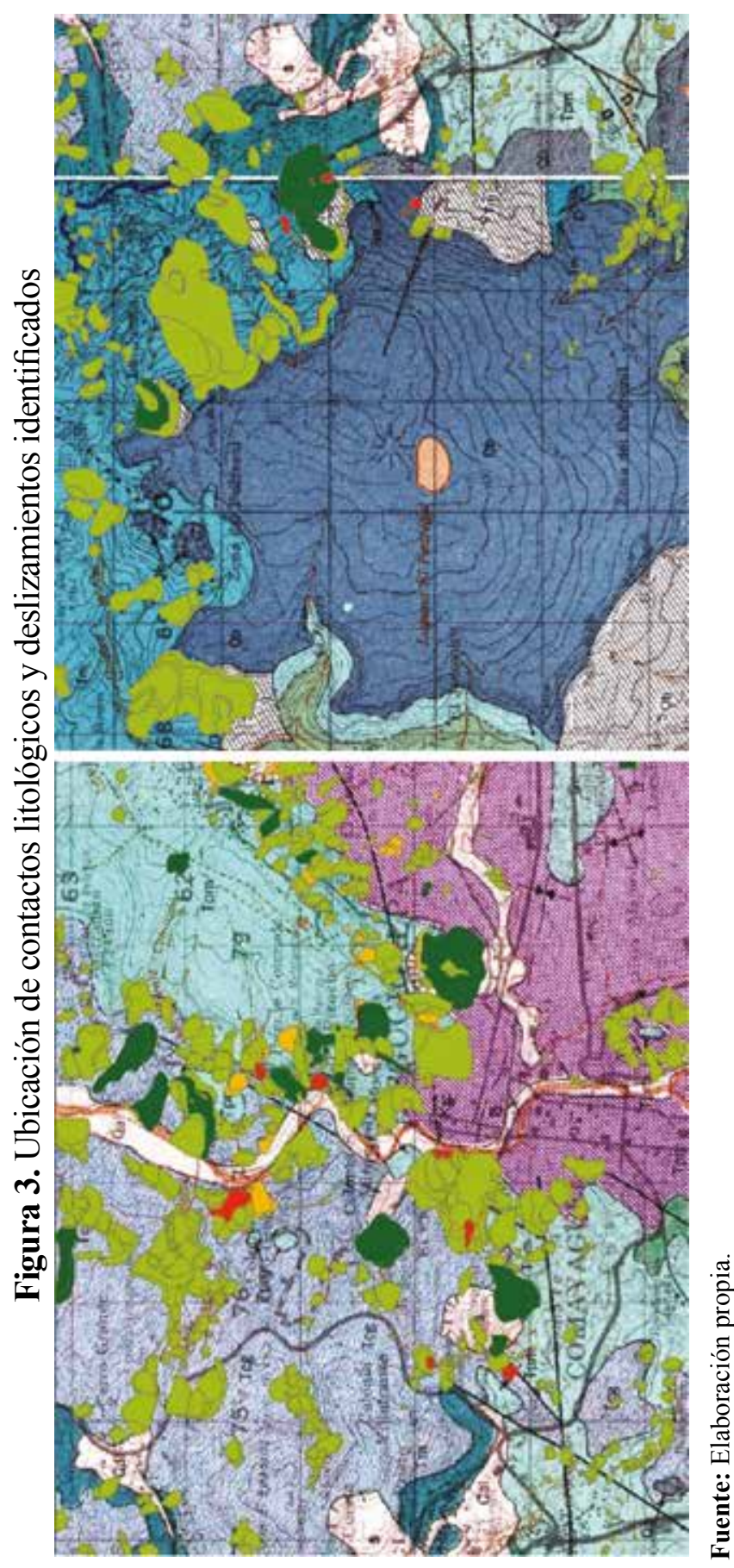


Adolfo Quesada-Román, Rigoberto Moncada-López, Jorge Antonio Paz-Tenorio, Eveling Espinoza-Jaime, Carmen Gutiérrez-Gutiérrez, Alex Castellón-Meyrat, Norwin Acosta-Galeano. Research on hillslope processes in Costa Rica, Honduras, Mexico and Nicaragua: teachings from the academy, cooperation agencies and public institutions

\section{Los estudios de movimientos de ladera en Honduras}

Dentro de Honduras, una cantidad considerable de los estudios detallados en el tema de deslizamientos se ha realizado en la ciudad capital, ya que su vulnerabilidad ha resultado en constantes pérdidas humanas y materiales por los movimientos de ladera. Desde este punto de vista, las investigaciones que sirven como punto de partida son los estudios desarrollados por la USGS como Harp et al. (2002), entre otros, y el Plan Maestro de JICA (2002), generados en los años posteriores al desastre del Huracán Mitch. Seguidamente, otros proyectos como DIPECHO por parte de PNUD (2010) y DesInventar (Rosales, 1998) han aportado al conocimiento de la ubicación y susceptibilidad de los deslizamientos.

Una comparación y validación interesante de estas investigaciones anteriores con el inventario desarrollado por JICA, es aquella con el Mapa de Amenaza por Remoción en Masa Ajustado por el Método Heurístico desarrollado por PNUD/GOAL/CI AMBIENTAL, con actualización en 2013. Un porcentaje considerable de los deslizamientos (aprox. 98\%) identificados en el mapa de inventario de 2014 coincide con las zonas de alta amenaza, determinadas por el mapa con el método heurístico para las zonas en común analizadas entre ambos estudios.

Aun así, es importante recalcar que se identificaron signos de deslizamientos en zonas que no se clasificaron como amenaza alta, sino media (Ver Figura 4). La razón de estas diferencias es la tendencia a generarse movimientos de ladera en lugares de pendientes bajas y moderadas (menores a 20 grados) debido a la presencia de suelos residuales de deslizamientos anteriores. Otro factor relacionado puede ser la erosión ocasionada por ríos al pie de las laderas. Asimismo, por diferencias en resolución de los datos, hubo algunas fallas en laderas de unas pocas decenas de metros cuadrados que el mapa de multi-amenaza no identificó. Por esta razón, el mapa de inventario se puede considerar un aporte relevante para mejorar la exactitud en determinar lugares sensibles a deslizamientos y, sobre todo, en definir la forma de los escarpes, cuerpos, etc.

Aun con un mapa de inventario que identifica cientos de objetos específicos, la información actual es insuficiente. Es indispensable una caracterización in situ, objeto por objeto, de todos los deslizamientos identificados. El proyecto "Assistancefor Strengthening and Capacity-building of Professional Techniques for the Control and Mitigation of Landslide in 
Adolfo Quesada-Román, Rigoberto Moncada-López, Jorge Antonio Paz-Tenorio, Eveling Espinoza-Jaime, Carmen Gutiérrez-Gutiérrez, Alex Castellón-Meyrat, Norwin Acosta-Galeano. Las investigaciones sobre movimientos de laderas en Costa Rica, Honduras, México y Nicaragua: enseñanzas desde la academia, las agencias de cooperación y las instituciones públicas

Tegucigalpa Metropolitan Area", desarrollado entre 2015-2016 por la Universidad Autónoma de Honduras con apoyo de JICA, ha realizado trabajos de este tipo y se espera continúen en el futuro cercano analizando en campo la mayor cantidad de lugares posibles (Kuwano et al., 2016). También es necesario pasar a fases posteriores del mapeo de deslizamiento, con mapas de susceptibilidad, amenazas y riesgo con objetos de alta resolución. Finalmente, es vital que este mapeo sea acompañado por cambios en la gestión de la planificación urbana, legislación territorial y de permisos de construcción. Por ejemplo, materiales como el manual para la evaluación del riesgo de la infraestructura, desarrollado por la Cooperación Suiza y PNUD (Suarez, 2013), permiten la evaluación del riesgo de un emplazamiento de una manera objetiva, en la medida de lo posible.

Figura 4. Topografías de deslizamiento en Tegucigalpa

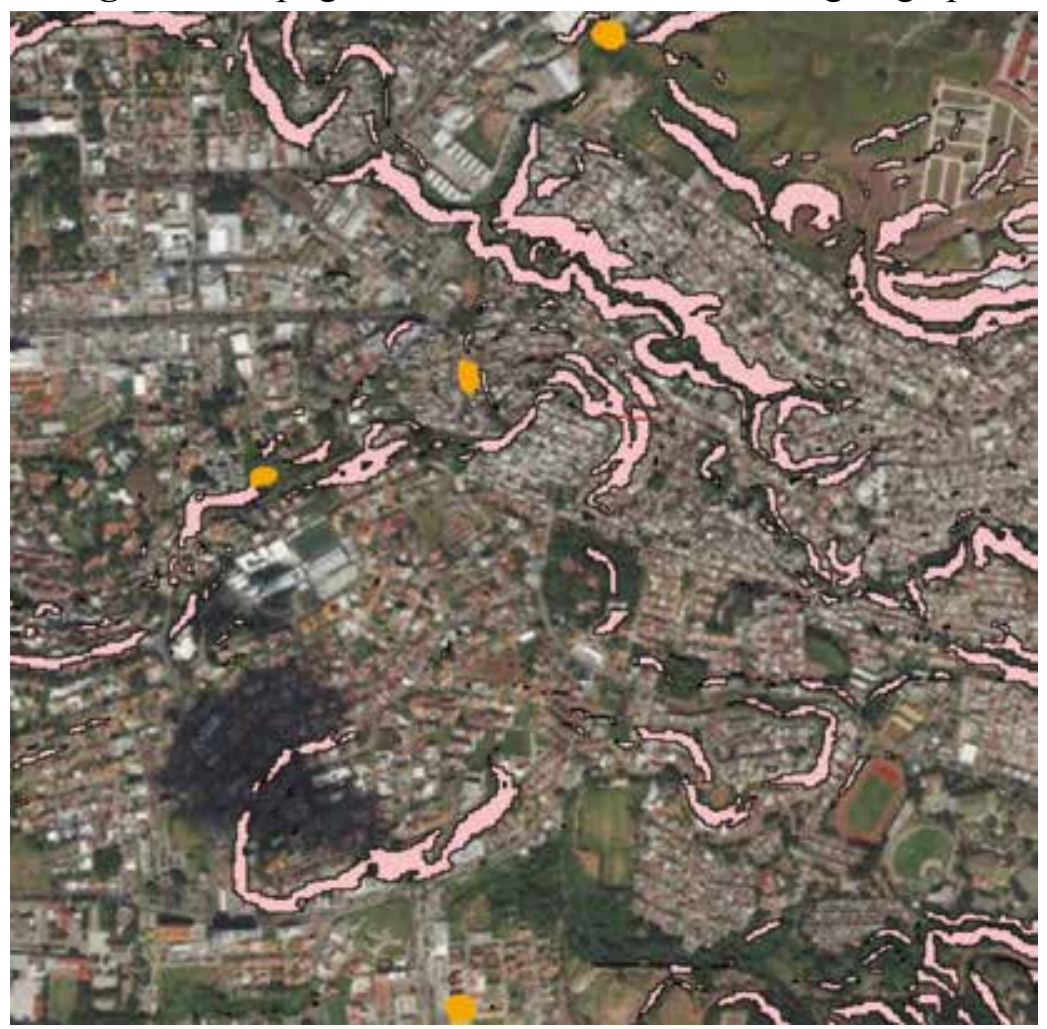

Fuente: CI AMBIENTAL (2013). La imagen muestra de Topografías de deslizamiento (dorado) que no coinciden con zonas de amenaza alta a movimientos de ladera (rosado) 
Adolfo Quesada-Román, Rigoberto Moncada-López, Jorge Antonio Paz-Tenorio, Eveling Espinoza-Jaime, Carmen Gutiérrez-Gutiérrez, Alex Castellón-Meyrat, Norwin Acosta-Galeano. Research on hillslope processes in Costa Rica, Honduras, Mexico and Nicaragua: teachings from the academy, cooperation agencies and public institutions

\section{Caso de México: Tuxtla Gutiérrez, Chiapas}

Chiapas es el estado más meridional de la República Mexicana. Presenta una gran amplitud en su composición geológica, diferencias altitudinales, climas y asociaciones vegetales. Los componentes sociales y económicos no son la excepción, siendo Centroamérica un territorio con el que mantiene gran afinidad tanto en los aspectos mencionados como en sus relaciones cotidianas. Tuxtla Gutiérrez, capital de la entidad chiapaneca, forma parte de uno de los 118 municipios que integran el estado. El municipio tiene una extensión de $334.61 \mathrm{~km}^{2}$, es decir, el $0.45 \%$ del territorio estatal (Gobierno de Chiapas, 2012).

Tuxtla Gutiérrez, como varios núcleos urbanos, históricamente, ha crecido de manera rápida y desordenada, siendo la infraestructura y su población las que resienten los efectos. Se localiza al sur de la República Mexicana, en las coordenadas $16^{\circ} 54^{\prime} 11^{\prime \prime}$ de latitud norte y $93^{\circ} 06^{\prime} 56^{\prime \prime}$ de longitud oeste, su altitud varía en las partes elevadas habitadas de 830 msnm a 550 en la plaza central. La mesa kárstica de Copoya ocupa poco más de un tercio de la superficie del municipio, se localiza al sur de la ciudad, y es precisamente el crecimiento urbano hacia sus flancos, lo que ha desatado la problemática de movimientos de ladera a partir del año 1995, cuando se documentan los primeros daños (Paz-Tenorio, 2017).

La mesa de Copoya está constituida por rocas calizas-areniscas del Eoceno que corresponden a la formación Lomut (INEGI 2006a; SGM 2006). Otros autores (Ferrusquía-Villafranca, et al. 2000; Membrillo-Ortega, 2006) la clasifican en el Eoceno medio como formación San Juan. Se formó en ambientes marinos someros de acuerdo a los estudios paleontológicos (Mullerried, 1957; Ferrusquía-Villafranca et al. 2000; AvendañoGil, 2005). Estas calizas-areniscas yacen sobre limolitas y areniscas del Eoceno inferior de la formación El Bosque (SGM 2006) que a su vez se sobreponen a lutitas y areniscas del Paleoceno correspondientes a la formación Soyaló (Ferrusquía-Villafranca et al. 2000).

Estas últimas unidades litológicas se encuentran ocultas en su mayor parte en los alrededores de la mesa por depósitos recientes conformados por grandes bloques de caliza desprendidos (Colegio de Ingenieros Geólogos de México, 2000) debido al intenso fracturamiento marginal, además de roca muy alterada, por lo que la ladera es inestable de origen. Esta geoforma presenta condiciones favorables para los desprendimientos, ya que el fracturamiento 
Adolfo Quesada-Román, Rigoberto Moncada-López, Jorge Antonio Paz-Tenorio, Eveling Espinoza-Jaime,

Carmen Gutiérrez-Gutiérrez, Alex Castellón-Meyrat, Norwin Acosta-Galeano. Las investigaciones sobre movimientos de laderas en Costa Rica, Honduras, México y Nicaragua: enseñanzas desde la academia, las agencias de cooperación y las instituciones públicas

marginal es abundante, y la naturaleza kárstica, sumada a la acción del agua, genera intensos procesos de disolución, lo que se manifiesta en la abundancia de cuevas, simas y abrigos rocosos (Grupo Espeleológico Vaxakmen, 2009). Los desprendimientos generan largos escarpes que Lugo-Hubp, et al. (2005) clasifican como circos, efecto de erosión remontante. Ante estos elementos condicionantes, actúan los elementos detonadores como las lluvias abundantes entre los meses de mayo a octubre, la intensa y frecuente sismicidad por encontrarse bajo la influencia de la zona de subducción de las placas tectónicas de Cocos y Norteamérica, además de la placa del Caribe; y finalmente, la constante y mal ejecutada actividad humana con la apertura de conjuntos habitacionales, deforestación y alteraciones a la geometría original de la pendiente.

Fisiográficamente, se ubica dentro de la Depresión Central (Mullerried, 1957), que se encuentra delimitada por la sierra Madre de Chiapas al sur y el Altiplano central al norte. La mesa de Copoya tiene una elevación que oscila de los 800 a los $900 \mathrm{msnm}$, encontrando al extremo noroeste el cerro Mactumatzá (cerro de las once estrellas en lengua zoque) que alcanza los $1.140 \mathrm{msnm}$. Rodeada de valles fluviales, hacia el norte se define el correspondiente al río Sabinal, en donde se registran altitudes promedio de $550 \mathrm{msnm}$, hacia el este se encuentra el río Grijalva, siendo esta la parte más baja, cuya altitud oscila entre los 400 y $410 \mathrm{msnm}$. Hacia el sur y oeste, el valle del río Suchiapa se encuentra a $450 \mathrm{msnm}$. De estos datos se observan desniveles que oscilan entre $\operatorname{los} 300$ a $400 \mathrm{~m}$, situación que induce a energías de relieve considerables (Lugo-Hubp, 1988).

\section{Metodología}

La aplicación de la heurística como método de aproximación resultó adecuada al contar con un vasto conocimiento documental y de campo de la zona, y compensar las limitantes de escala de la información cartográfica disponible. Esto representa un sólido sustento para la elaboración del mapa de Amenazas por Procesos de Remoción en Masa para Tuxtla Gutiérrez, Chiapas escala 1:85.000 (Paz-Tenorio et al., 2012). Por lo anterior, el método heurístico con base en mapas cualitativos propuesto, fue sin duda el adecuado. En 2015, se revisa el mapa y se decide mejorarlo, aplicando además el análisis multicrietrio, combinación también empleada por Muñiz-Jáuregui y Hernández-Madrigal (2012) en su mapa de susceptibilidad a Remoción en Masa en Puerto Vallarta, Jalisco, México. 
Adolfo Quesada-Román, Rigoberto Moncada-López, Jorge Antonio Paz-Tenorio, Eveling Espinoza-Jaime, Carmen Gutiérrez-Gutiérrez, Alex Castellón-Meyrat, Norwin Acosta-Galeano. Research on hillslope processes in Costa Rica, Honduras, Mexico and Nicaragua: teachings from the academy, cooperation agencies and public institutions

Al momento de jerarquizar las variables empleadas, se estableció que, bajo condiciones de pendiente, drenaje y vegetación semejantes entre las laderas norte y sur, debían ser determinantes las diferencias litológicas, en primer lugar, y edáficas en segundo término. Esto, debido a que la ladera que se encuentra al norte de la ciudad no presenta hasta el momento problemas de inestabilidad. Por lo tanto, las variables involucradas fueron: geología, edafología, desniveles, densidad de drenaje y uso del suelo y vegetación.

\section{Resultados}

Previamente al Mapa de Amenazas por Procesos de Remoción en Masa de Tuxtla Gutiérrez (Paz-Tenorio et al. 2011) (Figura 5), se obtuvo el mapa de Geoformas (2012, mejorado en 2015) (Figura 6), que caracteriza el relieve de acuerdo a la taxonomía propuesta por Priego et al. (2008), en donde se refieren a los depósitos de talud como "rampas de piedemonte". A este mapa se le adicionó la información disponible en el inventario de eventos, que suma 31 eventos.

Limitante importante fueron las escalas de las fuentes cartográficas, ya que la cartografía temática se encuentra a escala de 1:250.000; la topográfica disponible a escala 1:50.000 con el apoyo de la topográfica escala 1:20.000 (de reciente edición) permitió identificar deslizamientos antiguos en los flancos de la mesa kárstica de Copoya. Se compensó la diferencia de detalle entre las escalas de la cartografía temática y topográfica, esto a partir de la definición de la unidad mínima cartografiable (UMC), que depende de la metodología propuesta para cada mapa. Para el caso de la cartografía 1:250.000, un área de $4 \mathrm{~mm}$ por lado en el mapa, equivale a 1 $\mathrm{km}$ por lado en el terreno (INEGI, 2006b).

Sin embargo, considerando las celdas de $1 \mathrm{~km}$ por lado, en ocasiones había conflictos en su interpretación. A pesar de ello, este mapa fue retomado para el Atlas de Riesgos de Tuxtla Gutiérrez (H. Ayuntamiento Municipal, 2012). Al respecto, Lugo-Hubp (1988), menciona que este tipo de mapas elaborados a partir de figuras geométricas puede mantener este formato para su representación final, o afinarse a través de métodos de interpolación para el trazo de isolíneas. 
Adolfo Quesada-Román, Rigoberto Moncada-López, Jorge Antonio Paz-Tenorio, Eveling Espinoza-Jaime, Carmen Gutiérrez-Gutiérrez, Alex Castellón-Meyrat, Norwin Acosta-Galeano. Las investigaciones sobre movimientos de laderas en Costa Rica, Honduras, México y Nicaragua: enseñanzas desde la academia, las agencias de cooperación y las instituciones públicas

Figura 5. Mapa de Amenazas por Procesos de Remoción en Masa para Tuxtla Gutiérrez, Chiapas, México (2012)

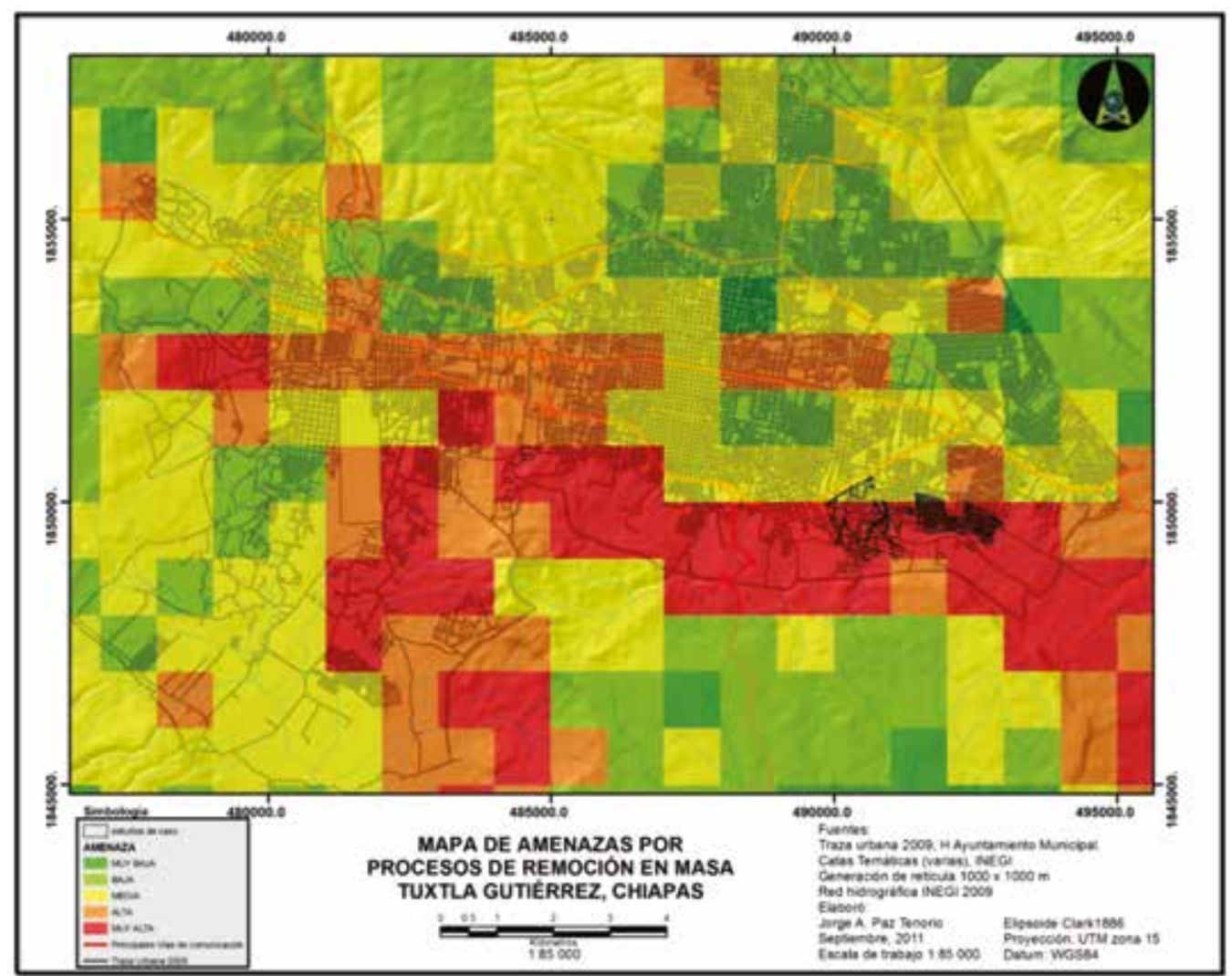


Adolfo Quesada-Román, Rigoberto Moncada-López, Jorge Antonio Paz-Tenorio, Eveling Espinoza-Jaime, Carmen Gutiérrez-Gutiérrez, Alex Castellón-Meyrat, Norwin Acosta-Galeano. Research on hillslope processes in Costa Rica, Honduras, Mexico and Nicaragua: teachings from the academy, cooperation agencies and public institutions

Figura 6. Inventario de Eventos y Geoformas para Tuxtla Gutiérrez, Chiapas, México (Paz-Tenorio, 2012)

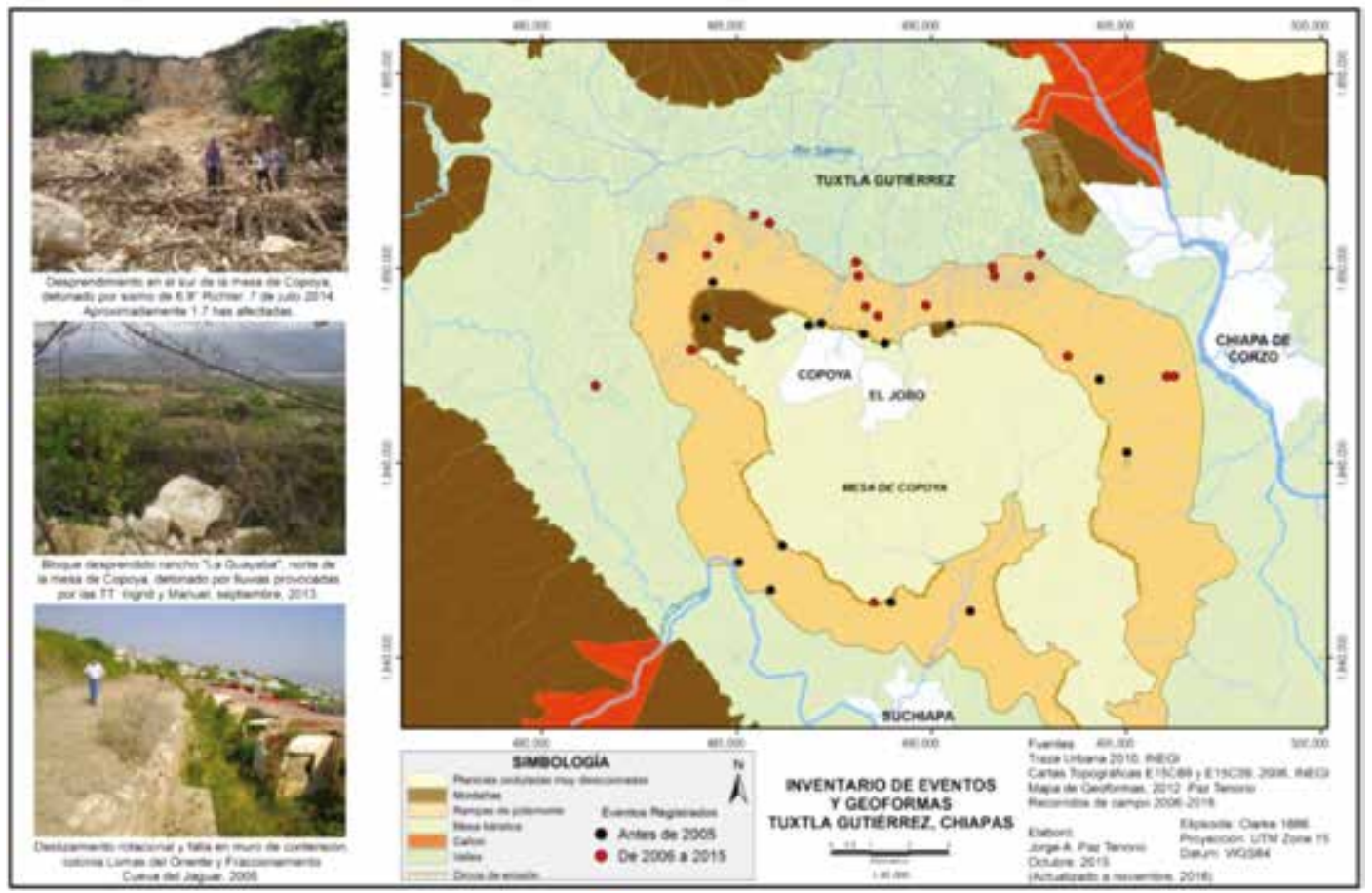

Fuente: Paz-Tenorio et al. (2017)

Para el mapa mejorado (Paz-Tenorio et al., 2017), a cada cuadro de la retícula se le generó un centroide para aplicar el proceso de interpolación y generar las isolíneas. Para las variables numéricas como densidad de drenaje y desniveles, se utilizaron los rangos definidos (número de intervalos iguales) por el software ArcMap (versión 9.3). Para las variables no cuantitativas como son geología, edafología y uso del suelo y vegetación, se utilizó el mismo método AHP, obteniendo valores numéricos para la representación cartográfica.

A través de métodos de interpolación, aplicando el método Kriging de la extensión Spatial Analyst, se logró perfeccionar el Mapa (Figura 7).

La jerarquización de las variables quedó como se muestra, apoyándose en el Proceso de Análisis Jerárquico (Analytic Hierarchy Process AHP) (Saaty, 1988): 
Adolfo Quesada-Román, Rigoberto Moncada-López, Jorge Antonio Paz-Tenorio, Eveling Espinoza-Jaime,

Carmen Gutiérrez-Gutiérrez, Alex Castellón-Meyrat, Norwin Acosta-Galeano. Las investigaciones sobre movimientos de laderas en Costa Rica, Honduras, México y Nicaragua: enseñanzas desde la academia, las agencias de cooperación y las instituciones públicas
1. Geología
2. Desniveles
3. Densidad de drenaje
4. Edafología
5. Uso del suelo y vegetación

Esta jerarquización de criterios se sustenta a partir de las investigaciones realizadas en la zona por especialistas, entre ellos, se han mencionado los trabajos de: Muciño-Porras, Domínguez-Salazar y Villalvazo-Báez (2005), Membrillo-Ortega (2006), Mora et al. (2007), Gobierno de Chiapas (2011); Espíritu (2012), y Secretaría de Gobierno (2015).

Figura 7. Mapa de Amenazas por Procesos de Remoción en Masa para Tuxtla Gutiérrez, Chiapas, México (2015)

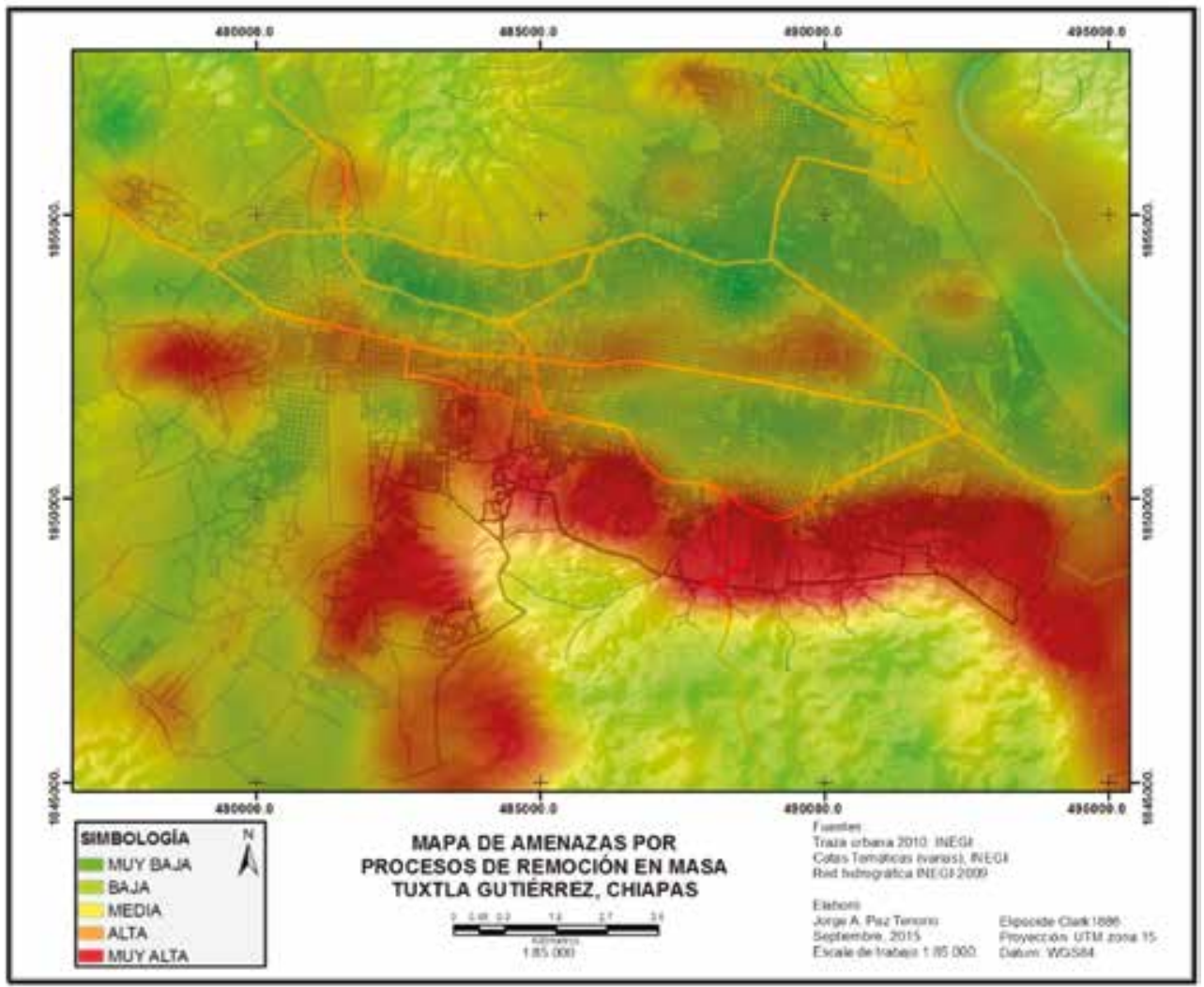

Fuente: Paz-Tenorio et al. (2015) 
Adolfo Quesada-Román, Rigoberto Moncada-López, Jorge Antonio Paz-Tenorio, Eveling Espinoza-Jaime, Carmen Gutiérrez-Gutiérrez, Alex Castellón-Meyrat, Norwin Acosta-Galeano. Research on hillslope processes in Costa Rica, Honduras, Mexico and Nicaragua: teachings from the academy, cooperation agencies and public institutions

En las variables no cuantitativas se obtuvieron los valores normalizados y un Índice de Consistencia (IC) y la Relación de Consistencia (RC) (este último de acuerdo a la dimensión de la matriz), inferiores al 10\% (Saaty, 1988), por lo que las ponderaciones son correctas. Este método también es empleado por Aceves et al. (2006); Borja-Baeza (2012); Celemín (2014) Hernández et al. (2013); Muñiz-Jáuregui y Hernández-Murillo (2012) y Murillo-García (2013), esta última, lo menciona como uno de los métodos aplicables.

Una vez refinado el método, se procedió a estimar los elementos expuestos. Para el caso de estudio de la ladera sur en Tuxtla Gutiérrez, se presentaron cinco niveles de amenaza en la zona, abarcando los siguientes porcentajes: Muy baja 5\%, Baja 27.1\%, Media 39.3\%, Alta $15.3 \%$ y Muy Alta $13.3 \%$, siendo esta última la que se distribuye en su mayor parte en los depósitos de talud, alrededor de la mesa de Copoya, lo que confirma su situación de máximo peligro ante movimientos de ladera. Se calcula una población expuesta de aproximadamente 76.975 habitantes $(14 \%$ del total) (537.102 habitantes en el área urbana) que residen tanto en la parte sur de la ciudad como en 30 localidades rurales asentadas en los flancos de la mesa de Copoya; se estiman poco más de 23.968 viviendas expuestas agrupadas en 1.041 manzanas.

\section{Los estudios de procesos de ladera en México}

Paz-Tenorio et al. (2011) realizan una revisión de casos ocurridos en Chiapas entre 2006 y 2011, en este documento, se registran eventos tanto en zonas rurales como urbanas e, inclusive, zonas de cultivo de café. Algunas instituciones como el Servicio Geológico Mexicano (SGM) (2016), han elaborado cartografía de peligros y/o riesgos a nivel estatal y municipal. El SGM reporta: atlas de peligros de Tamaulipas, Hidalgo, Querétaro, Tlaxcala, Puebla, Oaxaca, Tabasco y Chiapas, entre otros.

La Secretaría de Gobernación, a través del CENAPRED, en su portal de internet, al 31 de mayo de 2013, publicó un resumen de los Atlas Municipales de Peligros y Riesgos disponibles en el país para ese año (actualmente ya no está disponible esta información); en total eran 21 municipios de 14 estados los que disponen de esta importante herramienta en la gestión del riesgo. Instituto Nacional de Estadística y Geografía (INEGI) en 2011 reporta la existencia de 2.456 municipios en el país, por lo que, 
Adolfo Quesada-Román, Rigoberto Moncada-López, Jorge Antonio Paz-Tenorio, Eveling Espinoza-Jaime,

Carmen Gutiérrez-Gutiérrez, Alex Castellón-Meyrat, Norwin Acosta-Galeano. Las investigaciones sobre movimientos de laderas en Costa Rica, Honduras, México y Nicaragua: enseñanzas desde la academia, las agencias de cooperación y las instituciones públicas

con base al dato arriba mencionado, solamente al $0.8 \%$ del total nacional han publicado este documento. El estado de Veracruz destacaba a nivel nacional al tener la mayor cantidad de publicaciones referentes a Atlas de Riesgos para 5 municipios. Continúan: Quintana Roo con 3, Baja California Sur, Colima y Campeche con 2; finalmente, Baja California, Chihuahua, Sinaloa, Tamaulipas, Morelos, Guerrero, Oaxaca y Yucatán con 1. Todos disponibles en ese año a través de un sitio web.

En cuanto a bases de datos existentes, La RED, a través del Sistema de inventario de efectos de desastres DesInventar, permite el libre acceso a una base de registros de sucesos ocurridos en países de América Latina (excepto Brasil), Asia y África (http://www.desinventar.org/es/). Sin embargo, solo 4 casos relacionados con deslizamientos son reportados para Tuxtla Gutiérrez, Chiapas, cuando en el mapa de inventario elaborado por PazTenorio (2017), se disponen de 31 para el mismo municipio.

El Instituto Nacional de Estadística y Geografía publica un mapa de Susceptibilidad a Movimientos en Masa en la zona del Macizo Chiapaneco (INEGI, 2015), escala 1:500.000. En él se identifican 6.305 eventos, principalmente, a partir de la interpretación de ortofotografías e imágenes recientes obtenidas del software libre Google Earth. No se especifica cuántos ni cuáles fueron verificados durante recorridos de campo. Cabe destacar que el área analizada representa el $30 \%$ del territorio chiapaneco, y no abarca zonas en donde históricamente se han registrado diferentes y recurrentes PG, como en el caso de las montañas del norte y la ciudad de Tuxtla Gutiérrez, capital del estado.

\section{Caso de Nicaragua: Análisis y evaluación de la susceptibilidad por deslizamientos en San Juan del Río Coco}

En Nicaragua, los movimientos de laderas representan uno de los procesos geológicos de alta recurrencia, principalmente, en la estación lluviosa con precipitación máxima en los meses de junio y septiembre, que corresponde al $20 \%$ de los totales anuales. Estos eventos constituyen una amenaza en las vidas humanas, cultivos e infraestructura. La ocurrencia de terrenos inestables en Nicaragua obedece, exclusivamente, a factores condicionantes como la geología, el grado de pendiente y aspectos determinantes de la edad, naturaleza y calidad de las rocas y suelos de determinada área o territorio. Al respecto, es importante conocer las propiedades físico-mecánicas de los 
Adolfo Quesada-Román, Rigoberto Moncada-López, Jorge Antonio Paz-Tenorio, Eveling Espinoza-Jaime, Carmen Gutiérrez-Gutiérrez, Alex Castellón-Meyrat, Norwin Acosta-Galeano. Research on hillslope processes in Costa Rica, Honduras, Mexico and Nicaragua: teachings from the academy, cooperation agencies and public institutions

materiales, la estructura tectónica regional y local, aspectos como el patrón climático y de precipitación en los procesos de erosión - sedimentación, estabilidad de taludes, así como la actividad sísmica regional, semi-regional y local. En este sentido, este estudio propone una zonificación cartográfica de la susceptibilidad por inestabilidad de laderas, se centra principalmente en conocer y caracterizar la distribución espacial de los factores condicionantes y desencadenantes que influyen en el desarrollo de deslizamientos en el municipio y alrededores de San Juan del Río Coco.

San Juan del Río Coco se localiza en las coordenadas $13.5448^{\circ} \mathrm{N}$ y $86.1663^{\circ} \mathrm{W}$, en el departamento de Madriz, en el norte-centro de Nicaragua, su población es de 21.000 habitantes (INEC, 2000); se distingue por su relieve montañoso y escarpado, con elevaciones de fuertes pendientes de $1.492 \mathrm{msnm}$ (Cerro del Malacate), entre otras elevaciones que promedian un poco menos que $1.000 \mathrm{msnm}$. Este relieve es coherente con su geología, conformada por un dominio de rocas metamórficas (filitas y esquistos) y, localmente, por rocas graníticas acidas (Volcán Cerro El Malacate) e intrusivos básicos menores; además de lavas volcánicas al sur. Todas estas rocas son cortadas por lineamientos y fallas geológicas con direcciones noreste y este-oeste, que contribuyen con el relieve abrupto del terreno generando las condiciones para que se produzcan diferentes tipos de inestabilidades.

De las rocas volcánicas se han derivado principalmente Alfisoles, suelos arcillosos de color rojizo, ricos en óxidos de hierro y aluminio y pH ácidos aptos para el cultivo de café bajo sombra; el uso de suelo dominante en el paisaje. La meteorización de rocas graníticas y metamórficas en climas secos, en combinación con fuertes pendientes, ha dado origen a Entisoles e Inceptisoles, suelos poco desarrollados, susceptibles a erosión y no aptos para la agricultura, donde predominan bosques de pino o ciprés. La principal fuente hidrográfica del municipio corresponde al Río Coco que recorre el territorio en dirección oeste - este, los ríos de la zona, como el San Juan que cuenta con una longitud de $17.96 \mathrm{~km}$ con área de cuenca de $6,314 \mathrm{~km}^{2}$, es alimentado de nacientes y quebradas que bajan de los cerros, como La Alcantarilla, La Palma, El Bálsamo, San Antonio y Samarcanda, dando lugar a un patrón de drenaje dendrítico. Estos cursos de agua se descargan al Río Coco, el drenaje más importante que sirve de límite con el Municipio San Sebastián de Yalí. 
Adolfo Quesada-Román, Rigoberto Moncada-López, Jorge Antonio Paz-Tenorio, Eveling Espinoza-Jaime,

Carmen Gutiérrez-Gutiérrez, Alex Castellón-Meyrat, Norwin Acosta-Galeano. Las investigaciones sobre movimientos de laderas en Costa Rica, Honduras, México y Nicaragua: enseñanzas desde la academia, las agencias de cooperación y las instituciones públicas

\section{Metodología}

A raíz del desastre por el paso del Huracán Mitch (1998), se inició de manera sistemática el estudio de movimientos de laderas y se hizo evidente la necesidad de contar con una herramienta base en la prevención y mitigación del riesgo. Se desarrolló una base de datos desde el año 2004, que incluye sitios potenciales a movimientos de laderas en asentamientos, poblados y zonas potenciales expuestas a esta amenaza. Los sitios críticos representan un conjunto de entornos (peligros naturales, vulnerabilidad de las condiciones en infraestructuras, condiciones socio-económicas, jurídicas, entre otras), donde es necesario y urgente la aplicación de medidas inmediatas de mitigación para evitar las posibles pérdidas de vidas humanas y daños a la propiedad e infraestructura. Para actualizar anualmente esta base de datos, se realizan levantamientos de información de campo, tomando en cuenta la guía técnica para la elaboración de mapas de amenaza por inestabilidad de laderas (MET-ALARN-COSUDE, 2005), el monitoreo continuo de acumulados de lluvia en tiempo real y vigilancia sísmica por ocurrencia de lahares en las zonas volcánicas, son herramientas claves para el monitoreo y alerta oportuna a la población.

A nivel municipal se han logrado elaborar mapas de susceptibilidad (escala 1:10.000, 1:25.000 y 1:30.000) ante fenómenos de inestabilidad de laderas como herramientas de apoyo para definir líneas de acción en la planificación territorial. Estos mapas se basan en el análisis de los factores condicionantes y desencadenantes que tienen influencia en el área de estudio y tratan de representar el comportamiento futuro de las laderas de acuerdo a evidencias intrínsecas del terreno.

En el análisis enfocado al proyecto de San Juan del Río Coco se utilizó el Método de Superposición de Mapas con Índices Multiclases, incluido en las herramientas del Análisis Espacial de ArcGIS (Versión 10.2). A partir de la combinación de factores condicionantes, tales como pendiente y geología, se logró identificar las laderas más propensas a la ocurrencia de deslizamientos. La información de pendientes se derivó de las curvas de nivel del terreno con intervalo vertical de 20 metros, obtenidas de los mapas topográficos de INETER, año 1988, escala 1:50.000; y la geología procede de los mapas geológicos elaborados por Catastro e Inventario de los Recursos Naturales, año 1972, escala 1:50.000. La información fue incorporada a una base de datos geográfica utilizando el programa ArcGIS 
Adolfo Quesada-Román, Rigoberto Moncada-López, Jorge Antonio Paz-Tenorio, Eveling Espinoza-Jaime, Carmen Gutiérrez-Gutiérrez, Alex Castellón-Meyrat, Norwin Acosta-Galeano. Research on hillslope processes in Costa Rica, Honduras, Mexico and Nicaragua: teachings from the academy, cooperation agencies and public institutions

(Versión 10.2). Para cada factor condicionante se generó un mapa preliminar, agrupando la información por categorías y asignando pesos relativos de susceptibilidad a inestabilidad de laderas. Los mapas se elaboran en formato raster con un tamaño de celda de 20 metros. El mapa resultante muestra cuatro zonas o rangos de susceptibilidad (baja, media, alta y muy alta). Esto permitió caracterizar la distribución espacial de los factores condicionantes, así como mostrar las áreas relativamente seguras ante deslizamientos y zonas críticas propensas a deslizarse.

\section{Resultados}

El mapa de susceptibilidad a deslizamientos de Nicaragua, escala 1:750.000 (Ineter-BGR, año 2003) muestra que la mayoría de sitios de inestabilidad de laderas se concentran en el relieve de montaña de las regiones del centro, norte y la cadena volcánica del Pacifico. El área de estudio se ubica la cordillera de Dipilto de relieve montañoso, caracterizada por zonas de altas pendientes. La ocurrencia de procesos de inestabilidad de laderas en San Juan del Río Coco se debe, fundamentalmente, a factores condicionantes como litología, pendiente, topografía, estructura y forma del terreno y erosión hídrica.

El cambio e intensivo uso del suelo, precipitaciones de larga duración, sismicidad local y variabilidad climática, a largo plazo constituyen los principales elementos para la detonación de movimientos de laderas. En la cartografía realizada a escala 1:25.000, se identificaron en su mayoría deslizamientos de tipo superficial, rotacional y traslacional en suelos lateriticos y rocas filitas y esquistos con alto grado de meteorización y en zonas de pendientes abruptas mayores de $30^{\circ}$.

Sitios de derrumbes puntuales se concentran en cortes verticales de roca fracturada y en la mayoría de los casos meteorizada y zonas de pendientes abruptas, considerados como sitios peligrosos de amenaza alta, debido a que han causado daños especialmente a los tramos de carretera y caminos de acceso. Así mismo se identificaron zonas de derrumbe en sitios canteras, donde se da mucha remoción de material, de la capa vegetal, lo que facilita la erosión directa de la roca causando un peligro de inestabilidad de las, laderas.

Reptación o movimiento lento del suelo son bastante comunes en terrenos metamórficos que se evidencia por el desarrollo de caminos de 
vaca, cárcavas y aparición de pequeños, pero continuos escarpes en forma de graderíos. Las condiciones favorables son lomas desforestadas usadas como potreros, pendientes mayores de $60^{\circ}$, roca meteorizada e inclinación de la foliación siguiendo la pendiente, lo que las hace muy susceptibles a movimientos de laderas. En el Mapa de inventario por inestabilidad de laderas, estas áreas tienen un grado de amenaza de medio a bajo.

Se establecieron cuatro zonas de susceptibilidad (Cuadro 1 y Figura 7) en el municipio San Juan de Río Coco; las zonas de susceptibilidad alta a muy alta es donde se producen con mayor frecuencia y magnitud procesos de inestabilidades, están identificadas con colores rojo claro y oscuro en el mapa (Figura 8). Esta zona corresponde al 44,796\% del área y cubre zonas de laderas escarpadas con pendientes mayores de $20^{\circ}$, en rocas metamórficas altamente intemperizadas y fracturadas. La ocurrencia de deslizamientos es no menor del $50 \%$, en condiciones de lluvia intensa y posiblemente sismos importantes en pendiente mayores de $30^{\circ}$.

Zona de susceptibilidad media: representa el 39,77\% del área, está identificada en color naranja. Cubre las laderas con pendientes de $10^{\circ}$ a $20^{\circ}$. Es una zona de inicio de deslizamientos por la remoción de bloques, escarpes, acelerados por la lluvia o sismos.

En color amarillo están las zonas de susceptibilidad baja, presenta condiciones menos favorables para la ocurrencia de inestabilidades, predomina un relieve plano con pendientes bajas normalmente menor que $10^{\circ}$, corresponde a las zonas relacionadas con los valles de los ríos, sujetas a inundación y flujos torrenciales durante la lluvia. Por su ubicación entre zonas de alta y media susceptibilidad son propensas a los impactos de inestabilidades generadas sobre ellas. Es común encontrar acumulación de depósitos coluviales en la base de las laderas como evidencias de derrumbes o deslizamientos ocurridos. Por ello, debe ser considerada como zona de impacto y tener un tipo de regulación especial.

La zona de baja susceptibilidad comprende el 15,47\% del área. Representa zonas viables para emplazamiento de sitios urbanos e infraestructura de servicios públicos como escuelas, centros de salud y centros de albergues, siempre y cuando se realicen medidas correctivas, y se consideren sectores aledaños con susceptibilidad media a muy alta. 
Adolfo Quesada-Román, Rigoberto Moncada-López, Jorge Antonio Paz-Tenorio, Eveling Espinoza-Jaime, Carmen Gutiérrez-Gutiérrez, Alex Castellón-Meyrat, Norwin Acosta-Galeano. Research on hillslope processes in Costa Rica, Honduras, Mexico and Nicaragua: teachings from the academy, cooperation agencies and public institutions

Cuadro 1. Características y clase de susceptibilidad a inestabilidad de laderas

\begin{tabular}{|l|l|l|c|}
\hline \multicolumn{1}{|c|}{$\begin{array}{c}\text { Factor } \\
\text { condicionante }\end{array}$} & \multicolumn{1}{|c|}{ Agrupación } & Susceptibilidad & $\begin{array}{c}\text { Peso } \\
\text { relativo }\end{array}$ \\
\hline Pendiente (en & 010 & Baja & 1 \\
grados) & $10-20$ & Media & 2 \\
& 2030 & Alta & 3 \\
& $>30$ & Muy Alta & 4 \\
\hline Geología & PTif (Plioceno Terciario Intrusivo félsico) & Baja & 1 \\
& PTim (Plioceno Terciario Intrusivo máfico) & Baja & 1 \\
& PTii (Plioceno Terciario Intrusivo intermedio) & Baja & 1 \\
& PTm (Paleozoico Terciario Metamórfico) & Alta & 3 \\
& Tomm (Terciario Oligoceno Mioceno Matagalpa) & Alta & 3 \\
\hline
\end{tabular}


Adolfo Quesada-Román, Rigoberto Moncada-López, Jorge Antonio Paz-Tenorio, Eveling Espinoza-Jaime, Carmen Gutiérrez-Gutiérrez, Alex Castellón-Meyrat, Norwin Acosta-Galeano. Las investigaciones sobre movimientos de laderas en Costa Rica, Honduras, México y Nicaragua: enseñanzas desde la academia, las agencias de cooperación y las instituciones públicas

Figura 8 y 9: Mapa de Inventario y susceptibilidad por deslizamientos del poblado de San Juan de Río Coco y alrededores
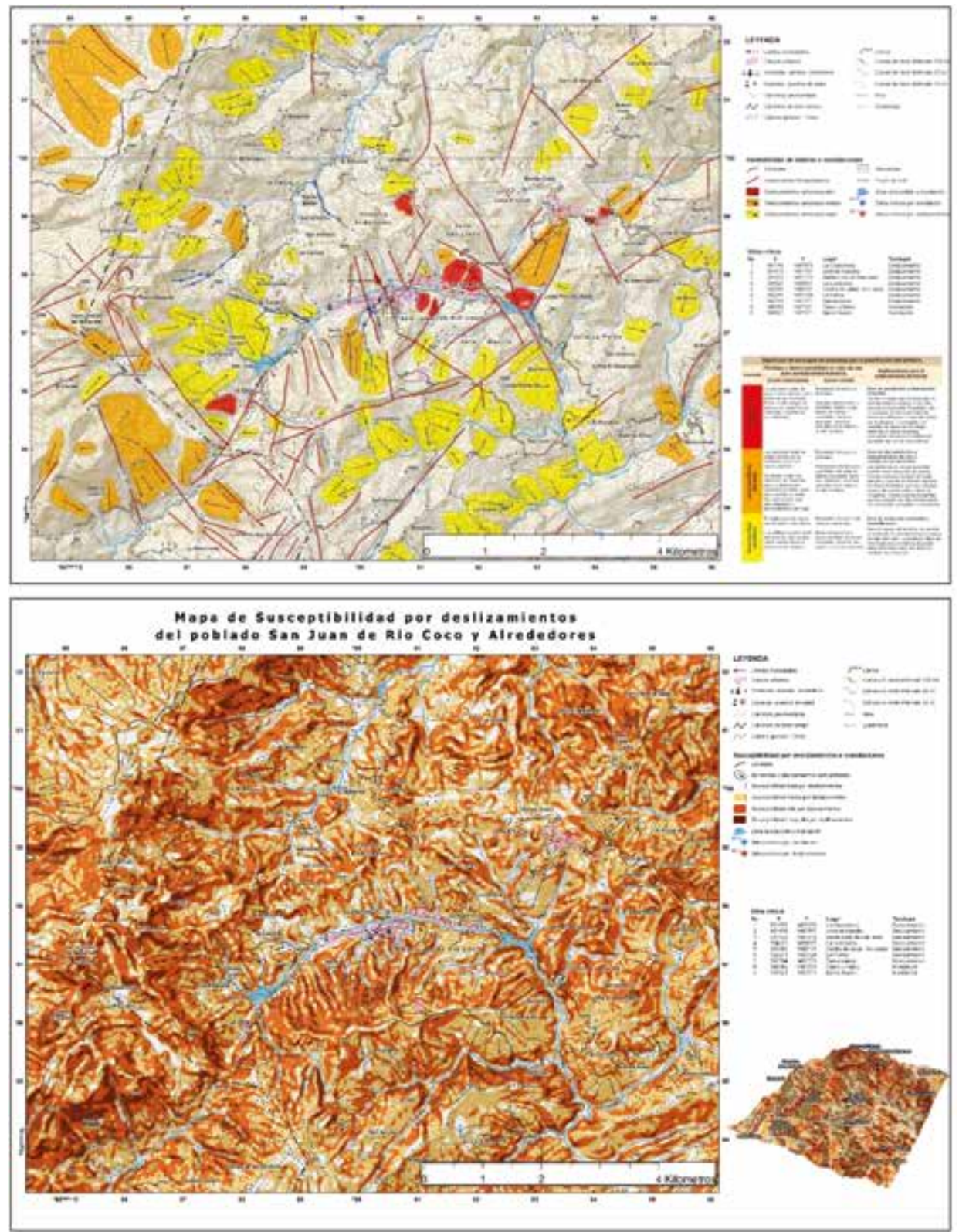

Fuente: Ineter-BGR, 2003 
Adolfo Quesada-Román, Rigoberto Moncada-López, Jorge Antonio Paz-Tenorio, Eveling Espinoza-Jaime, Carmen Gutiérrez-Gutiérrez, Alex Castellón-Meyrat, Norwin Acosta-Galeano. Research on hillslope processes in Costa Rica, Honduras, Mexico and Nicaragua: teachings from the academy, cooperation agencies and public institutions

\section{Monitoreo y control de deslizamientos en Nicaragua}

Por sus características geográficas y geológicas, Nicaragua presenta una alta susceptibilidad a los movimientos de laderas. A finales del año 1998, después del paso del Huracán Mitch y la ocurrencia del deslave del volcán Casita al occidente del país, con más de 2.000 víctimas, se inician de manera sistemática estudios de deslizamientos en Nicaragua, a nivel nacional para identificar y evaluar las áreas inestables. En las regiones Centro, Norte y la cordillera volcánica existen registros históricos de grandes deslizamientos que han cobrado vidas humanas y materiales, relacionados con lluvias fuertes y otros eventos meteorológicos y geológicos que determinan y provocan una gran variedad de acontecimientos peligrosos como deslizamientos, lahares, caída de rocas, flujos detritos y lodos, entre otros. De cara a la eventualidad de estos fenómenos se ha desarrollado un análisis y obtención de mapas pronósticos de procesos de deslizamientos en todo el país, que sirva como una herramienta base en la óptica de prevención y reducción del riesgo a desastre por movimientos de laderas, y que contribuya en la toma de decisiones de la planificación y el ordenamiento territorial.

Es muy importante señalar que la escala a la que se han realizado estos estudios, debe de ampliarse con la finalidad de definir mejores zonas potenciales y su posible reactivación, así como monitoreo continuo de estos procesos, con la finalidad de tener información detallada para tomar medidas de prevención y planificación del uso del terreno en el municipio. Existe una base de datos creada en 1999 a través de un Sistema de Información Geográfica (SIG). La información de la base de datos se recopilo, mediante proyectos de mapeo realizados en el tema de susceptibilidad de laderas y el reconocimiento geológico en sitios donde han ocurrido deslizamientos, principalmente en el norte, centro y a lo largo de la cadena de volcanes activos del país. Un total de 2.003 sitios de deslizamientos se habían registrados al año 2005.

Para actualizar anualmente la base de datos nacional de los sitios por deslizamientos, principalmente, en áreas, asentamientos y poblados expuestos a esta amenaza, se realiza levantamiento de información en campo, haciendo uso de mapas topográficos (escala 1:50.000, 1988) y fotografías aéreas (escalas: 1:40.000/1:30.000; 2004, 1970), obtenida de la Dirección de Geodesia y Cartografía del Instituto Nicaragüense de Estudios 
Territoriales (INETER). En época lluviosa (meses de mayo a octubre), se realiza un monitoreo de los acumulados de lluvias en tiempo real de estos sitios susceptibles a deslizamientos, a través de imágenes satelitales, en las diferentes regiones del país, y con apoyo en los dos últimos años de ICDF y Universidad de Taiwán quienes proporcionan imágenes satelitales para el análisis de sitios inestables. Así mismo, se utilizan datos de lluvia actualizados cada 30 minutos con satelitales de la NOAA/NESDIS. El uso de herramientas web como la Plataforma integrada de Advertencia contra Todo Riesgo, Análisis y Evaluación de Riesgos (Pacific Disaster Center, 2011) y el monitoreo de la vigilancia sísmica son bases claves en el monitoreo oportuno de movimientos de laderas. Una limitante es la carencia de cartografía a detalle (50.000 o mayor).

\section{Discusión de resultados y conclusiones}

A partir de los estudios de caso por país y la disponibilidad de información para cada uno de ellos, se realizó un cuadro comparativo (Cuadro 2), en el cual se plasman los casos, áreas estudiadas, los insumos y herramientas, así como los productos obtenidos en términos de movimientos de ladera. 
Adolfo Quesada-Román, Rigoberto Moncada-López, Jorge Antonio Paz-Tenorio, Eveling Espinoza-Jaime, Carmen Gutiérrez-Gutiérrez, Alex Castellón-Meyrat, Norwin Acosta-Galeano. Research on hillslope processes in Costa Rica, Honduras, Mexico and Nicaragua: teachings from the academy, cooperation agencies and public institutions

Cuadro 2: Comparación de los estudios de caso: insumos, herramientas y productos

\begin{tabular}{|c|c|c|c|}
\hline CASO & AREA/ UNIDAD & $\begin{array}{c}\text { INSUMOS Y } \\
\text { HERRAMIENTAS }\end{array}$ & PRODUCTO \\
\hline COSTA RICA & $\begin{array}{l}\text { Cuenca Alta Río General } \\
9.584^{\circ} \text { y } 9.211^{\circ} \text { Lat } \mathrm{N} \\
-83.818^{\circ} \text { y }-83.479^{\circ} \text { Lon } \mathrm{W} \\
1.5 \mathrm{Km}^{2}\end{array}$ & $\begin{array}{l}\text { Base de Datos } \\
\text { Desinventar } \\
\text { Mapas Topográficos } \\
1: 50000,1: 25.000 . \\
\text { Fotografías Aéreas } \\
1: 25000(2005)\end{array}$ & $\begin{array}{l}\text { Mapa de Peligros } \\
\text { a Procesos de } \\
\text { Ladera de la } \\
\text { Cuenca Alta del } \\
\text { Río General } \\
\text { 1:25000 }\end{array}$ \\
\hline HONDURAS & $\begin{array}{l}\text { Zona Metropolitana } \\
\text { Tegucigalpa- } \\
\text { Comayagûela } \\
14.160 \text { y } 13.974^{\circ} \text { Lat N } \\
-87.300^{\circ} \text { y }-87.120^{\circ} \text { Lon } \mathrm{W} \\
380 \mathrm{Km}^{2}\end{array}$ & $\begin{array}{l}\text { Fotografías Aéreas } \\
\text { 1:9.000 } \\
\text { Mapa topográfico } \\
: 12000 \\
\text { Mapa Geológico } \\
\text { 1:50 } 000 \\
\text { Google Earth } \\
\text { SIG y recorridos de } \\
\text { campo }\end{array}$ & $\begin{array}{l}\text { Mapa de } \\
\text { Deslizamientos } \\
\text { De Tierra de } \\
\text { Tegucigalpa } \\
\text { 1:40 } 000\end{array}$ \\
\hline MÉXICO & \begin{tabular}{|l|} 
Zona Urbana de Tuxtla \\
Gutiérrez, Chiapas \\
$16.784^{\circ}$ y $16.702^{\circ}$ Lat N \\
$-93.192^{\circ}$ y $-93.023^{\circ}$ \\
Lon W \\
$185 \mathrm{Km}^{2}$
\end{tabular} & $\begin{array}{l}\text { Base de Datos } 2006 \text { a } \\
\text { la actualidad } \\
\text { Mapa Topográfico } \\
1.50000 \text { y } 1: 20.000 \\
\text { Mapas Geológicos, } \\
\text { Edafológicos y } \\
\text { Vegetación } 1: 250.000 \\
\text { SIG y recorridos de } \\
\text { campo }\end{array}$ & $\begin{array}{l}\text { Mapa de } \\
\text { Amenazas por } \\
\text { Procesos de } \\
\text { Remoción en } \\
\text { Masa de Tuxtla } \\
\text { Gutiérrez, Chiapas } \\
\text { 1:85000 }\end{array}$ \\
\hline NICARAGUA & \begin{tabular}{|l|} 
San Juan del Río Coco \\
$13.587^{\circ}$ y $13.504^{\circ}$ Lat N \\
$-86.220^{\circ}$ y $-86.111^{\circ}$ Lon W \\
$120 \mathrm{Km}^{2}$
\end{tabular} & $\begin{array}{l}\text { Base de Datos } 2004 \text { a } \\
\text { la Actualidad } \\
\text { Mapas Topográficos y } \\
\text { Geológicos 1:50.000 } \\
\text { Guía Técnica }\end{array}$ & $\begin{array}{l}\text { Mapa de Inventarío } \\
\text { y Susceptibilidad } \\
\text { por Deslizamientos } \\
\text { 1:25000 }\end{array}$ \\
\hline
\end{tabular}

Este estudio logra unir experiencias desde la Academia, Organizaciones No Gubernamentales y las Instituciones Públicas encargadas de la producción de cartografía y estudios para la gestión del riesgo de movimientos de ladera en Costa Rica, Honduras, México y Nicaragua. Es la primera vez que se hace un esfuerzo de este tipo entre dichos países, el cual nace a partir del Segundo Congreso Centroamericano de Movimientos de Ladera realizado en Julio de 2016 en Tegucigalpa. 
Adolfo Quesada-Román, Rigoberto Moncada-López, Jorge Antonio Paz-Tenorio, Eveling Espinoza-Jaime,

Carmen Gutiérrez-Gutiérrez, Alex Castellón-Meyrat, Norwin Acosta-Galeano. Las investigaciones sobre movimientos de laderas en Costa Rica, Honduras, México y Nicaragua: enseñanzas desde la academia, las agencias de cooperación y las instituciones públicas

Este trabajo refleja el estado actual de los estudios de los movimientos en masa, que junto con las inundaciones, es uno de los riesgos naturales que más afecta la región mesoamericana debido a sus características tectónicas, geológicas, climatológicas, y modificaciones antrópicas acaecidas, especialmente, en el último siglo, sobre todo con en el cambio del uso del suelo y el favorecimiento de procesos erosivos que llevan con el desarrollo de procesos de remoción en masa cada vez más frecuentes y peligrosos para la población.

El estudio da una visión general y regional de los alcances que tienen dichos estudios, muchas veces centrados en zonas de recurrente afectación por estos procesos, lo que sirve para mitigar el impacto de estos en el futuro, pero además da las pautas para desarrollar estas mismas metodologías en otras regiones de América Latina, dependiendo de los insumos con los que se cuenta, donde los movimientos de ladera también son una problemática ligada con los impactos antrópicos, y las variaciones de la geodinámica tanto interna como externa.

\section{Referencias}

Aceves-Quesada, F., López-Lanco, J., Martín del Pozzo, A.L. (2006). Determinación de peligros volcánicos aplicando técnicas de evaluación multicriterio y SIG en el área del Nevado de Toluca, centro de México. Revista Mexicana de Ciencias Geológicas, 23(2), 113-124.

Alcaldía Municipal del Distrito Central-AMDC. (2017) Portal de Mapas $A M D C$. [online] amdc.giscloud.com. Recuperado de http://amdc. giscloud.com/ [Fecha de acceso: 1 Feb. 2017].

Alcántara-Ayala, I. (2000). Landslides: deslizamientos o movimiento del terreno? Definición, clasificaciones y terminología. Investigaciones Geográficas: Boletín del Instituto de Geografía-UNAM, 41, 7-25.

Alcántara-Ayala, I. (2002). Geomorphology, natural hazards, vulnerability and prevention of natural disasters in developing countries, Geomorphology, 47: 107-124.

Anderson, M.G., Holcombe, E., Blake, J.R., Ghesquire, F.G., Holm-Nielsen, N., Fisseha, T. (2011). Reducing Landslide Risk in Communities: Evidence from the Eastern Caribbean. Applied Geography, 31(2): 590-599. DOI: 10.1016/j.apgeog.2010.11.001. 
Adolfo Quesada-Román, Rigoberto Moncada-López, Jorge Antonio Paz-Tenorio, Eveling Espinoza-Jaime, Carmen Gutiérrez-Gutiérrez, Alex Castellón-Meyrat, Norwin Acosta-Galeano. Research on hillslope processes in Costa Rica, Honduras, Mexico and Nicaragua: teachings from the academy, cooperation agencies and public institutions

Avendaño-Gil, M. J. (2005). Importancia paleontológica de la Mesa de Copoya. En: VI Aniversario Grupo Espeleológico Vaxakmen. Tuxtla Gutiérrez, Chiapas.

Bird, P. (2003). An updated digital model of plate boundaries, Geochemistry, Geophysics, Geosystems, 4(3): 1-52.

Caballero, E. (2010). Planificación del Territorio Urbano en Honduras: Entre la Acción Pública y de Mercado. Revista Postgrados UNAH, 4(1), 68-79.

Carías, A., García, D., Orbera, L. \& Cabrera, M. (2015). Correlación entre las Anomalías Morfométricas y los Deslizamientos en la Ciudad de Tegucigalpa. Revista Milímetro / Universidad Politécnica de Ingeniería, 1, 83-87.

CI AMBIENTAL S.A.S. (2013). Producto No.2 Actualización del Mapa de Amenazas por deslizamientos de la Ciudad de Tegucigalpa. (Informe Final y Mapa. Programa de las Naciones Unidas para el Desarrollo-PNUD y GOAL). Proyecto Honduras.

Borja-Baeza, R.C. (2012). Determinación de peligros volcánicos aplicando técnicas de evaluación multicriterio y SIG en el área del Nevado de Toluca, centro de México. (Tesis doctoral).

Celemín, J.P. (2014). El proceso analítico jerárquico en el marco de la evaluación multicriterio: un análisis comparativo. Geografía y Sistemas de Información Geográfica (GEOSIG), 6, 47-63.

Colegio de Ingenieros Geólogos de México, A. C. (2000). Estudio geológico ambiental del área donde se ubica el Zoológico regional "Miguel Álvarez del Toro" (ZOOMAT), de Tuxtla Gutiérrez, Estado de Chiapas. México. (Informe técnico).

Cruden, D.M. \& Varnes, D.J. (1996). Landslides Types and Processes. En Landslides: Investigation and Mitigation, edit. Por Keith A. Turner y Robert L. Schuster, 36-75. Washington: National Academy Press.

Denyer, P., Montero, W. y Alvarado, G.E. (2003). Atlas tectónico de Costa Rica. Editorial de la Universidad de Costa Rica. San José, Costa Rica. 79 pp.

Denyer, P. y Alvarado G.E. (2007). Mapa geológico de Costa Rica. Escala 1:400 000. San José, Costa Rica: Librería Francesa.

Devoli, G., Morales, A., \& Høeg, K. (2007). Historical landslides in Nicaragua-collection and analysis of data. Landslides, 4(1), 5-18. 
Adolfo Quesada-Román, Rigoberto Moncada-López, Jorge Antonio Paz-Tenorio, Eveling Espinoza-Jaime, Carmen Gutiérrez-Gutiérrez, Alex Castellón-Meyrat, Norwin Acosta-Galeano. Las investigaciones sobre movimientos de laderas en Costa Rica, Honduras, México y Nicaragua: enseñanzas desde la academia, las agencias de cooperación y las instituciones públicas

Ebert, A. and Kerle, N. (2008). Urban Social Vulnerability Assessment Using Object-Oriented Analysis of Remote Sensing and GIS Data. A Case Study for Tegucigalpa, Honduras. The International Archives of Photogrammetry, Remote Sensing and Spatial Sciences 37(B7): 1306-http://www.isprs.org/proceedings/XXXVII/congress/7 pdf/7_WG-VII-7/02.pdf

Espinoza-Jaime, E., Dávila-Lorente, I., Acosta-Galeano, N., CastellónMeyrat, A. y Gutiérrez-Gutiérrez, C. (2014). Actualización del Mapa de sitios críticos por deslizamientos en Nicaragua. INETER. Espíritu Tlatelpa, G. (2012). Movimientos de masas y sus implicaciones en el ordenamiento urbano. En: El Sudcaliforniano; Ciencia, Innovación y Tecnología para el desarrollo de México. 4,102. La Paz BCS. México. 22 de abril.

Ferrusquía-Villafranca, I., Applegate, S.P. \& Espinosa-Arrubarrena, L. (2000). First Paleogenesis elachifauna of the middle American-Caribbean Antillean region, La Mesa de Copoya, west-central Chiapas, Mexico-Geologic setting. Revista Mexicana de Ciencias Geológicas, 17(1), 1-23.

Gares, P. A., Shermany, D. \& Nordstrom, K. F. (1994). Geomorphology and Natural Hazards, Geomorphology, 10, 1-18. DOI: 10.1016/0169555X (94)90004-3.

Gobierno de Chiapas. (2012). Carta Geográfica del Estado de Chiapas. Escala 1:500 000.

Gobierno de Chiapas. (2011). Dictamen de Identificación de Riesgos (Colonia Lomas del Oriente). Instituto de Protección Civil para el Manejo Integral de Riesgo de Desastres Tuxtla Gutiérrez, Chiapas. 18 de enero. pp 26.

Grupo Espeleológico Vaxakmen. (2009). Proyecto Copoya, Chiapas. Memorias del IXI Congreso Nacional Mexicano de Espeleología. Puebla, Puebla, México. Febrero. 67-70 pp.

Grupo de Mapeo de Deslizamientos de Tierra de Tegucigalpa (TLMG). (2014). Manual Para Elaboración de InventaRio de Deslizamientos de Tierra. Caso de aplicación: Ciudad de Tegucigalpa. Agencia de Cooperación Internacional del Japón (JICA). Tegucigalpa. ISBN: 978-99926-701-1-8 
Adolfo Quesada-Román, Rigoberto Moncada-López, Jorge Antonio Paz-Tenorio, Eveling Espinoza-Jaime, Carmen Gutiérrez-Gutiérrez, Alex Castellón-Meyrat, Norwin Acosta-Galeano. Research on hillslope processes in Costa Rica, Honduras, Mexico and Nicaragua: teachings from the academy, cooperation agencies and public institutions

Harp, E., Reid, M., McKenna, J. \& Michael, J. (2009). Mapping of hazard from rainfall-triggered landslides in developing countries: Examples from Honduras and Micronesia. Engineering Geology, 104, 295-311.

Harp, E.L., Held, M.D., Castañeda, M.R., McKenna, J.P. \& Jibson, R.W. (2002). Landslide hazard map of Tegucigalpa, Honduras. U.S. Geological Survey Open-File Report 02-219. 9 pp.

Hernández-Moreno, G., Borja-Baeza, R.C., Garnica-Peña, R.J. y Alcántara-Ayala, I. (2013). Susceptibilidad a Procesos de Remoción en Masa. En: Atlas de Factores de Riesgo de la Cuenca de Motozintla, Chiapas.

Highland, L.M. \& Bobrowsky, P. (2008). The Landslide Handbook-A Guide to Understanding Landslides. Circular 1325. Virginia: Reston, Geological Survey.

Hirota, K. \& Kamiya, S. (2014). Re-evaluation of Landslide Caused by Hurricane Mitch 1998, Tegucigalpa Honduras. In: Landslide Science for a Safer Geoenvironment: Volume 3: Targeted Landslides. Proceedings of World Landslide Forum 3, 2-6 June 2014, Beijing, 393-400 pp.

Huggett, R. (2007). Fundamentals of Geomorphology. Routledge. New York, Estados Unidos.

Hungr, O., Leroueil, S. \& Picarelli, L. (2013). The Varnes classification of landslide types, an update. Landslides, 11(2), 167-194.

Instituto Nicaragüense de Estudios Territoriales (INETER). (2009). Estudio sismológico y geológico Asociado a la actividad sísmica marzo de 2009, Municipio San Juan de Río Coco, Departamento de Madriz.40 pp.

Instituto Nicaragüense de Estudios Territoriales (INETER). (2001). Amenazas Naturales de Nicaragua. Managua. 288 páginas.

Instituto Nicaragüense de Estudios Territoriales (INETER). (2008). Monitoreo satelital de acumulados de lluvia. Recuperado de: http://deslizamientos.ineter.gob.ni/

Instituto Geográfico Nacional (IGN). (1987). Mapa Geológico de Tegucigalpa, Lepaterique y San Buenaventura. Tegucigalpa, Honduras. 1:50,000. 
Adolfo Quesada-Román, Rigoberto Moncada-López, Jorge Antonio Paz-Tenorio, Eveling Espinoza-Jaime,

Carmen Gutiérrez-Gutiérrez, Alex Castellón-Meyrat, Norwin Acosta-Galeano. Las investigaciones sobre movimientos de laderas en Costa Rica, Honduras, México y Nicaragua: enseñanzas desde la academia, las agencias de cooperación y las instituciones públicas

Instituto Hondureño de Ciencias de la Tierra (IHCIT). (2014). Estudio Hidrogeoquímico de la parte alta de la cuenca del Río Choluteca. Subproducto 2.4, Actualización del inventario de recursos de aguas subterráneas. Universidad Autónoma de Honduras. Proyecto Fondo de Adaptación. 159 pp.

Instituto Hondureño de Ciencias de la Tierra (IHCIT). (2012). Atlas Climático y de Gestión de Riesgo de Honduras. Universidad Nacional Autónoma De Honduras. 161 pp.

Instituto Hondureño de Ciencias de la Tierra (IHCIT). (2011). Cálculo de Umbrales para la Ciudad de Tegucigalpa. Universidad Nacional Autónoma De Honduras. Convenio SERNA/UNAH. Proyecto Fondo de Adaptación. 40 pp.

Instituto Nacional de Estadística y Geografía (INEGI).. (1984). Carta Topográfica E15C69 Tuxtla Gutiérrez. Escala 1:50 000. Proyección UTM zona 15. México.

Instituto Nacional de Estadística y Geografía (INEGI). (2006a). Carta Geológica E1511. Escala 1:250 000. Proyección UTM zona 15. México, archivo digital.

Instituto Nacional de Estadística y Geografía (INEGI). (2006b). Metodología para la actualización de la cartografía de Uso del Suelo y Vegetación, Serie V. Mimeog. Aguascalientes, México.

Instituto Nacional de Estadística y Geografía (INEGI).. (2011). Censo General de Población y Vivienda 2010. Tabulados Básicos. Consulta en línea. www3.inegi.org.mx/sistemas/TabuladosBasicos/Default. aspx? $\mathrm{c}=27302 \& \mathrm{~s}=$ est.

Instituto Nacional de Estadística y Geografía (INEGI).. (2015). Mapa de Susceptibilidad del Fenómeno de Movimiento en Masa del Macizo de Chiapas. Disponible en: http://www3.inegi.org.mx/sistemas/biblioteca/ficha.aspx?upc $=702825209384$

Japanese International Cooperation Agency (JICA). (2002). The study on flood control and landslide prevention in the Tegucigalpa metropolitan area of the Republic of Honduras. Prepared by Pacific Consultants International and Nikken Consultants, Inc., Tegucigalpa, Honduras.

Kreft, S., Eckstein, D. \& Melchior, I. (2016). Global Climate Risk Index 2017. Who Suffers Most From Extreme Weather Events? Weather-related Loss Events in 2015 and 1996 to 2015. Germanwatch. Briefing Paper. 
Adolfo Quesada-Román, Rigoberto Moncada-López, Jorge Antonio Paz-Tenorio, Eveling Espinoza-Jaime, Carmen Gutiérrez-Gutiérrez, Alex Castellón-Meyrat, Norwin Acosta-Galeano. Research on hillslope processes in Costa Rica, Honduras, Mexico and Nicaragua: teachings from the academy, cooperation agencies and public institutions

Kuwano, T., Hara, T., Tsukamoto, S., Inaoka, M. \& Suzuki, H. (2016). JICA Project for strengthening and capacity building of landslide control and mitigation in Honduras. Proceedings of Second Central American and Caribbean Landslide Congress, 18-20 July 2016, Tegucigalpa, Honduras.7-12 pp.

LA RED (Red de Estudios Sociales en Prevención de Desastres en América Latina) - Corporación OSSO. (2015). DesInventar: Sistema de inventario de efectos de desastres de Costa Rica entre 1970 y 2013. Ciudad de Panamá, Panamá.

Lugo-Hubp, J. (1988). Elementos de Geomorfología aplicada (Métodos cartográficos). IG/UNAM. México D.F.

Lugo- Hubp, J., Zamorano-Orozco, J. J., Capra, L., Inbar, M. y AlcántaraAyala, I. (2005). Los procesos de remoción en masa en la Sierra Norte de Puebla, octubre de 1999: Causas y efectos. Revista Mexicana de Ciencias Geológicas, 22(2), 212-228.

Lugo-Hubp, J. (2011). Diccionario Geomorfológico. Instituto de Geografía, UNAM. Ciudad de México, México. 479 pp.

Membrillo-Ortega, H. (2006). Geología de la meseta de Copoya. Mimeog. Tuxtla Gutiérrez. 16 pp.

MET-ALARN INETER-COSUDE. (2005). Recomendaciones Técnicas para la Elaboración de Mapas de Amenazas por Inestabilidad de Laderas. Managua, Nicaragua. 88 páginas.

Mora-Chaparro, J.C., Carrera, M., García-Diego, A.M., Escobedo, S., Figueroa, H.N., Hernández, V.M. y Sol, L.L. (2007). Reporte de observaciones geológicas en la ciudad de Tuxtla Gutiérrez, Chiapas. Informe técnico. Instituto de Protección Civil para el Manejo Integral de Riesgos de Desastre. pp 26.

Muciño-Porras, J. J., Domínguez -Salazar, F.F. y Villalvazo- Báez, M. (2005). Informe técnico del problema de deslizamiento en las colonias Lomas del Oriente y la Cueva del Jaguar. Informe. Tuxtla Gutiérrez, Chiapas. pp 11.

Murillo-García, F. G. (2013). Análisis y Cartografía de Riesgo de Desastre por Procesos de Remoción en Masa en el Municipio de Pahuatlán, Puebla. Tesis de Maestría, 2013. UNAM. 198 pp.

Mullerried, F.K.G. (1957). Geología de Chiapas. Tuxtla Gutiérrez. Gobierno de Chiapas. 
Adolfo Quesada-Román, Rigoberto Moncada-López, Jorge Antonio Paz-Tenorio, Eveling Espinoza-Jaime,

Carmen Gutiérrez-Gutiérrez, Alex Castellón-Meyrat, Norwin Acosta-Galeano. Las investigaciones sobre movimientos de laderas en Costa Rica, Honduras, México y Nicaragua: enseñanzas desde la academia, las agencias de cooperación y las instituciones públicas

Muñiz-Jáuregui, J.A. y Hernández-Madrigal, V.M. (2012). Zonificación de procesos de remoción en masa en Puerto Vallarta, Jalisco, mediante combinación de análisis multicriterio y método heurístico. Revista Mexicana de Ciencias Geológicas, 29(1), 103-114.

Pacific Disaster Center. (2011). Plataforma Integrada de Advertencia contra Todo Riesgo, Análisis y Evaluación de Riesgos. Recuperado dehttp://emops.pdc.org/

Paz-Tenorio, J. A., Gómez-Ramírez, M., González-Herrera, R. y Domínguez-Salazar, F.F. (2011). Los procesos de remoción en masa; génesis, efectos y limitaciones en el crecimiento urbano de Tuxtla Gutiérrez, Chiapas, México. Revista Geográfica de América Central, Número Especial EGAL 2011, 1-18

Paz-Tenorio, J. A. (2012). La inestabilidad de laderas y la construcción social del riesgo; tres casos en el sur de Tuxtla Gutiérrez, Chiapas. (Tesis de Maestría) Universidad de Ciencias y Artes de Chiapas. Noviembre, 2012. $160 \mathrm{pp}$.

Paz-Tenorio, J.A., González-Herrera, R., Gómez-Ramírez, M. y VelascoHerrera J.A. (2017). Metodología para elaborar Mapas de Amenazas por Procesos de Remoción en Masa, análisis del caso ladera sur de Tuxtla Gutiérrez, Chiapas. Investigaciones Geográficas. Boletín del Instituto de Geografía/UNAM 92: 1-16. DOI: dx.doi.org/10.14350/ rig.52822

Paz-Tenorio, J. A. (2017). Procesos Gravitacionales y la Construcción Social del Riesgo en Tuxtla Gutiérrez, Chiapas, México. (Tesis Doctoral). Universidad de Ciencias y Artes de Chiapas, México. 153 pp. Peña-Monné, J. L. (1997). Cartografía Geomorfológica: Básica y Aplicada. Geoforma Ediciones. Logroño, España.

Programa de las Naciones Unidas para el Desarrollo-PNUD. (2010).Informe de Línea Base: Proyecto DIPECHO VII UNDP. 14 Barrios Vulnerables a Deslizamientos y Sismos en la Ciudad de Tegucigalpa.

Priego, A., Bocco, G., Mendoza, M. y Garrido, A. (2001). Especificaciones técnicas que deberán contener la cartografia y la clasificación para la elaboración de los inventarios de suelos. NOM-023-RECNAT-2001, Secretaría del Medio Ambiente y Recursos Naturales D.O.F. $10 / \mathrm{dic} / 01$ 
Adolfo Quesada-Román, Rigoberto Moncada-López, Jorge Antonio Paz-Tenorio, Eveling Espinoza-Jaime, Carmen Gutiérrez-Gutiérrez, Alex Castellón-Meyrat, Norwin Acosta-Galeano. Research on hillslope processes in Costa Rica, Honduras, Mexico and Nicaragua: teachings from the academy, cooperation agencies and public institutions

Quesada-Román, A. (2015). Implicaciones en la gestión del riesgo de desastres y ambiente en el Valle Central en los últimos treinta años (1985-2015). Informe Final. Vigesimoprimer Informe Estado de la Nación en Desarrollo Humano Sostenible (2014).

Quesada-Román, A. (2016). Peligros geomorfológicos: inundaciones y procesos de ladera en la cuenca alta del río General (Pérez Zeledón), Costa Rica. Maestría en Geografía con énfasis en Geografía Ambiental. Posgrado en Geografía. Universidad Nacional Autónoma de México.

Quesada-Román, A. (2017). Geomorfología Fluvial e Inundaciones en la Cuenca Alta del Río General, Costa Rica. Anuário do Instituto de Geociências - UFRJ 40(2): 278-288. DOI: http://dx.doi. org/10.11137/2017_2_278_288

Rogers, R.D. \& O’Conner, E.A. (1993). Mapa Geológico de Honduras: Hoja de Tegucigalpa (segunda edición). Instituto Geográfico Nacional, Tegucigalpa, Honduras. 1:50.000.

Rosales, C. (1998). Asistencia técnica para la evaluación de los efectos del huracán Mitch en Honduras, 17nov - 8dic, 1998. Reporte. Red de Estudios Sociales en Prevención de Desastres en América Latina - LA RED/ Comité Permanente de Contingencias - COPECO

Saaty, T. L. (1988). The Analytic Hierarchy Process; Planning, Priority Setting Resource Allocation. New York, McGraw-Hill.287 pp.

Sato, G. (2015). Investigation and Mitigation of Landslide in Republic of Honduras as Japan's International Cooperation Program. Journal of the Japanese Landslide Society, 52(4), 1-7.

Secretaría de Gobernación. (SEGOB). (2013). Estados y municipios que a mayo de 2013 disponen de Atlas de Peligros y Riesgos, elaborados con apoyo de Fondo para la Prevención de Desastres Naturales (FOPREDEN) coordinado por SEGOB y del programa HABITAT de SEDESOL. México. Recuperado de www.atlasnacionalderiesgos.gob.mx/index. php?option $=$ com_content\&view $=$ article\&id $=120 \& I t e m i d=213$.

Secretaría de Gobierno (SEGOB) (2015). Visita técnica de seguimiento al proceso de inestabilidad de laderas que afecta a las Colinas Lomas del Oriente y Cueva del Jaguar, municipio de Tuxtla Gutiérrez, Chiapas, nota técnica. México: Centro Nacional de Prevención de Desastres, México. pp 26. 
Adolfo Quesada-Román, Rigoberto Moncada-López, Jorge Antonio Paz-Tenorio, Eveling Espinoza-Jaime,

Carmen Gutiérrez-Gutiérrez, Alex Castellón-Meyrat, Norwin Acosta-Galeano. Las investigaciones sobre movimientos de laderas en Costa Rica, Honduras, México y Nicaragua: enseñanzas desde la academia, las agencias de cooperación y las instituciones públicas

Selby, M.J. (1985). Earth’s Changing Surface. Oxford: Oxford University Press.

Servicio Geológico Mexicano. (2006). Carta Geológico Minera E15-11. Escala 1:250 000. Proyección UTM zona 15 México D.F.

ServicioGeológicoMexicano.(2016).CartografiaAmbiental.Recuperadode:http://www. sgm.gob.mx/index.php?option=com_content\&task=view\&id=32\&Itemid=39

Sterlacchini, S., Ballabio, C., Blahut, J., Masetti, M. \& Sorichetta, A. (2011). Spatial agreement of predicted patterns in landslide susceptibility maps. Geomorphology, 125(1): 51-61. DOI: 10.1016/j. geomorph.2010.09.004.

Suarez, G. (2013). Manual para la Evaluación de Riesgo del Emplazamiento y del Medio Construido para Edificios, Viviendas y Lotificaciones. COSUDE.COPECO.PNUD. ISBN: 978-99926-821-4-2

Varnes, D. J. (1978). Slope Movement Types and Processes. In Schuster, R.

L. and Krizek, R. J. (eds) Landslides-Analyses and Control Special Report 176. National Academy of Sciences. Washington, D.C., 11-34. Yamagishi, H. \& Moncada, R. (2017). Landslide Recognition and Mapping using Aerial Photographs and Google Earth. Landslide Dynamics: ISDR-ICL Landslide Interactive Teaching Tools (LITT) (en proceso de publicación).

Yamagishi, H. (2014). Integrated Disaster Prevention Maps and Landslide Hazard Maps using GIS -Examples of Shikoku and Central America Honduras-.Journal of the Japanese Landslide Society, 51(2), 24-29. Yamagishi, H., Yagi, H. \& Sato, G. (2014). Landslide Hazard Mapping of Tegucigalpa, Honduras-Capacity Building by JICA-JSPS Project of 2012-2014. Proceedings of World Landslide Forum 3, 2-6 June 2014, Beijing, pp. 712-715.

WP/WLI (International Geotechnical Societies UNESCO Working Party on World Landslide Inventory). (1990). A suggested method for reporting a landslide, Bulletin of the International Association of Engineering Geology, 41, 5-12. DOI: 10.1007/BF02590201. 